\title{
Points of Bounded Height on Equivariant Compactifications of Vector Groups, I
}

\author{
ANTOINE CHAMBERT-LOIR ${ }^{1}$ and YURI TSCHINKEL ${ }^{2}$ \\ ${ }^{1}$ Institut de mathématiques de Jussieu, Boite 247, 4, place Jussieu, F-75252 Paris Cedex 05, \\ France.e-mail: chambert@math.jussieu.fr \\ ${ }^{2}$ Department of Mathematics, U.I.C., Chicago, IL 60607-7045, U.S.A. \\ e-mail: yuri@math.uic.edu
}

(Received: 12 March 1999; accepted in final form: 12 November 1999)

\begin{abstract}
We prove asymptotic formulas for the number of rational points of bounded height on certain equivariant compactifications of the affine plane.
\end{abstract}

Mathematics Subject Classifications (2000). $11 \mathrm{G} 35$ (14J20, 14G05, 14G40).

Key words. height zeta function, rational points, equivariant compactification, Poisson formula, Manin's conjecture.

\section{Introduction}

Let $X$ be a smooth projective algebraic variety defined over a number field $F$ and $X(F)$ the set of rational points of $X$. Let $\mathcal{L}$ be a metrized ample line bundle and

$$
H_{\mathcal{L}}: X(F) \rightarrow \mathbf{R}_{>0}
$$

the associated exponential height (cf. $[15,18])$. We are interested in the asymptotic behaviour of the counting function

$$
N(U, \mathcal{L}, B):=\#\left\{x \in U(F) \mid H_{\mathcal{L}}(x) \leqslant B\right\}
$$

as $B \rightarrow \infty$, where $U \subset X$ is some Zariski open subset. Batyrev and Manin [1] and Peyre [13] described a conjectural framework for such asymptotics for varieties with ample (or, more generally, effective) anticanonical class (see also [6, 15] and references therein). In particular, it is expected that for appropriate $X$ and Zariski open subsets $U \subset X$,

$$
N\left(U, K_{X}^{-1}, B\right)=\frac{\Theta^{*}(X)}{r !} B(\log B)^{r}(1+o(1)),
$$

as $B \rightarrow \infty$. Here $K_{X}^{-1}$ is the metrized anticanonical line bundle on $X$, $r=\operatorname{rk} \operatorname{Pic}(X)-1$ and $\Theta^{*}(X)$ is a product of a Tamagawa type number $\tau\left(K_{X}\right)$ (which depends on the metrization), a rational number $\alpha^{*}(X)$ defined in terms of the cone of effective divisors $\Lambda_{\mathrm{eff}}(X)$ and the order $|\operatorname{Br}(X) / \operatorname{Br}(F)|$ of the nontrivial part 
of the Brauer group. There is a similar description for arbitrary polarizations $\mathcal{L}$ (Batyrev and Tschinkel, cf. [2]).

These conjectures have been proved for flag varieties ([6]), toric varieties ([2]), and toric bundles induced from torsors ([21]). The proofs use a precise combinatorial description of all geometric and arithmetic invariants of the varieties: line bundles, metrizations of the line bundles etc. (for example, in terms of lattices, cones and fans). Such a description is possible because representations of reductive groups are rigid (don't admit deformations). Consequently, the corresponding varieties don't have moduli.

The only other known approach to asymptotics of rational points on algebraic varieties is the classical circle method in analytic number theory. Varieties which can be treated by this method do admit moduli. However, one of the drawbacks is that so far it works only for varieties which are complete intersections of small degree $d$ in projective spaces $\mathbf{P}^{n}$ of large dimension (very roughly, $n \gg 2^{d}$ ). In particular, these complete intersections have Picard group Z. E. Peyre (cf. [16]) has extended the formalism of the circle method to complete intersections in other varieties (for example, toric varieties). Once the necessary estimates will be established, this should provide examples of asymptotics for varieties with moduli and with $\operatorname{Pic}(X)$ of higher ranks. As a reference to the circle method let us mention the papers by H. Davenport, R. Heath-Brown, C. Hooley on smooth cubic hypersurfaces (cf. [5, 9, 10]), by B. Birch and by W. Schmidt on general complete intersections (cf. [3, 20]).

In this paper we prove asymptotics of rational points of bounded height on varieties which admit moduli and which at the same time are closely related to (nonreductive) linear algebraic groups. More precisely, we consider smooth equivariant compactifications of the additive group $\mathbf{G}_{a}^{n}$. For $n=2$ it can be shown that all such compactifications are obtained as blow-ups of $\mathbf{P}^{2}$, or Hirzebruch surfaces in points which are fixed under the action of $\mathbf{G}_{a}^{2}$. Notice that a variety (even $\mathbf{P}^{2}$ ) may admit non-isomorphic structures as an equivariant compactification of $\mathbf{G}_{a}^{n}$. A similar 'minimal model program' of equivariant compactifications of $\mathbf{G}_{a}^{n}$ is a nontrivial problem already for $n=3$ (see [8]).

In this paper we study in detail the example of a blow-up of $\mathbf{P}^{2}$ in $r \mathbf{Q}$-rational points which are all contained in the line at infinity $\mathbf{P}^{1}$ (with the equation $x_{0}=0$ ). The moduli space of such surfaces $X$ is $\mathcal{M}_{0, r}$. It is easy to see that $X$ is a smooth projective equivariant compactification of $\mathbf{G}_{a}^{2}$ with $\operatorname{Pic}(X)=\mathbf{Z}^{r+1}$, trivial Brauer group and a simplicial cone of effective divisors $\Lambda_{\mathrm{eff}}(X)$. The constant $\alpha^{*}(X)$ is equal to $1 /\left(3 \cdot 2^{r}\right)$. Denote by $U \simeq \mathbf{G}_{a}^{2} \subset \mathbf{P}^{2}$ the complement to $x_{0}=0$. Then for $\operatorname{Re}(s) \gg 0$, the series

$$
Z\left(U, K_{X}^{-1}, s\right)=\sum_{\mathbf{x} \in U(\mathbf{Q})} H_{K_{X}^{-1}}(\mathbf{x})^{-s}
$$

is absolutely and uniformly convergent and defines a holomorphic function. One of the main results of this paper is the following: 
THEOREM 1. There exists a function $h$ which is holomorphic in the domain $\operatorname{Re}(s)>5 / 6$ such that

$$
Z\left(U, K_{X}^{-1}, s\right)=\frac{h(s)}{(s-1)^{r+1}} \quad \text { and } \quad h(1)=\alpha^{*}(X) \tau\left(K_{X}\right) \neq 0 .
$$

A standard Tauberian theorem implies that $X$ satisfies Peyre's refinement of Manin's conjecture:

COROLLARY 2. We have the following asymptotic formula:

$$
N\left(U, K_{X}^{-1}, B\right)=\frac{\alpha^{*}(X) \tau\left(K_{X}\right)}{r !} B(\log B)^{r}(1+o(1)),
$$

as $B \rightarrow \infty$.

In fact, we will prove asymptotics for every $\mathcal{L}$ on $X$ such that its class is contained in the interior of $\Lambda_{\text {eff }}(X)$. We will also give estimates for the growth of the function $h(s)$ in vertical strips in the neighborhood of $\operatorname{Re}(s)=1$. It is well known that this implies a more precise asymptotic expansion for the counting function $N(U, \mathcal{L}, B)$, see Corollary 4.17 .

We will address the compactifications of $\mathbf{G}_{a}^{n}$ (with $n>2$ ) in subsequent papers.

\section{Geometry}

\subsection{GENERALITIES}

Let $G$ be an algebraic group and $X$ a smooth projective variety with an action of $G$. We denote by $\operatorname{Pic}^{G}(X)$ the (Abelian) group of isomorphism classes of $G$-linearized line bundles on $X$ (cf. [12], Chap. 1, Section 3, Def. 1.6). We shall say that the variety $X$ is an equivariant compactification of $G$ if $X$ has an open dense subset $U$ which is equivariantly isomorphic to $G$. Well known examples are given by toric varieties which are equivariant compactifications of tori (algebraic groups isomorphic to $\mathbf{G}_{m}^{n}$ over the algebraic closure of $F$, where $\mathbf{G}_{m}=\operatorname{Spec}\left(F\left[x, x^{-1}\right]\right)$ is the multiplicative group scheme).

In this paper, we are interested in equivariant compactifications of $\mathbf{G}_{a}^{n}$, where $\mathbf{G}_{a}=\operatorname{Spec}(F[x])$ is the additive group scheme (we may call them addic varieties ${ }^{\star}$ ).

Notice that a variety can be an equivariant compactification of a group $G$ in many non-isomorphic ways, as the following example shows.

Example. The projective plane $\mathbf{P}^{2}$ is an equivariant compactification of $\mathbf{G}_{a}^{2}$ in (essentially) two non-isomorphic ways. One of the possible actions is the standard translation action, fixing a line $\mathbf{P}^{1}$ at infinity. All 1-parameter subgroups are lines.

ॠScherzhafter Vorschlag von Yu. I. Manin, Weihnachten 1998. 
The other action has exactly one fixed point and generic 1-parameter subgroups are conics (cf. [8] for more details, esp. Proposition 3.2).

We quote from [8] the following general geometrical facts about equivariant compactifications of additive groups.

PROPOSITION 1.2. Let $X$ be a smooth projective equivariant compactification of $\mathbf{G}_{a}^{n}$ and $D=X \backslash \mathbf{G}_{a}^{n}$ the boundary.

(1) The boundary $D$ is a pure codimension 1 subvariety.

(2) The Picard group $\operatorname{Pic}(X)$ is freely generated by the irreducible components $D_{0}, \ldots, D_{r}$ of $D$.

(3) The closed cone of effective divisors $\Lambda_{\mathrm{eff}}(X) \subset \operatorname{Pic}(X)_{\mathbf{R}}$ is given by

$$
\Lambda_{\mathrm{eff}}(X)=\bigoplus_{k=0}^{r} \mathbf{R}_{\geqslant 0}\left[D_{k}\right]
$$

(4) There exist integers $n_{k}>1$ such that the anticanonical class is given by

$$
\left[K_{X}^{-1}\right]=\sum_{k=0}^{r} n_{k}\left[D_{k}\right]
$$

\subsection{BLOW-UPS}

The basic example of an equivariant compactification of $\mathbf{G}_{a}^{n}$ is the projective space $\mathbf{P}^{n}$, with $\mathbf{G}_{a}^{n}$ acting on $\mathbf{P}^{n}=\operatorname{Proj}\left(F\left[x_{0}, \ldots, x_{n}\right]\right)$ by translation:

$$
\left(\left(t_{1}, \ldots, t_{n}\right),\left(x_{0}: \cdots: x_{n}\right)\right) \mapsto\left(x_{0}: x_{1}+t_{1} x_{0}: \cdots: x_{n}+t_{n} x_{0}\right)
$$

which stabilizes the 'hyperplane at infinity' given by the equation $x_{0}=0$. In this paper we consider blow-ups of the projective space $\pi: X \rightarrow \mathbf{P}^{n}$ in a subscheme $Z \subset \mathbf{P}^{n}$ of pure codimension $\geqslant 2$, which is contained in this hyperplane $Z_{0}$. We denote by $\mathcal{I}_{Z}$ the ideal sheaf of $Z$ in $\mathbf{P}^{n}$ so that the blow-up is defined by the formula $X=\operatorname{Proj}\left(\bigoplus_{j} \mathcal{I}_{Z}^{j}\right)$. As $Z \subset Z_{0}$ is fixed by $\mathbf{G}_{a}^{n}$, the universal property of the blow-up implies that the action of $\mathbf{G}_{a}^{n}$ on $\mathbf{P}^{n}$ lifts uniquely to an action on $X$.

The geometry of blow-ups of arbitrary subschemes can be very complicated. We shall assume that $\mathcal{I}_{Z}$ is the product of $r$ ideals $\mathcal{I}_{Z_{k}}(1 \leqslant k \leqslant r)$, where the $Z_{k}$ are integral subschemes of the hyperplane at infinity in $\mathbf{P}^{n}$. The universal property of blow-ups says that $X$ is the universal scheme mapping to $\mathbf{P}^{n}$ on which $\mathcal{I}_{Z}$ becomes invertible. An easy lemma in commutative algebra implies that on $X$, the $\mathcal{I}_{Z_{k}}$ are themselves invertible. (Blowing up a product of ideals is the universal way to make these ideals invertible; it is the same as successively blowing up the ideal of $Z_{1}$, then the inverse image of the ideal of $Z_{2}$, etc.) In particular, $\pi$ factors as $X \rightarrow X_{k} \rightarrow \mathbf{P}^{n}, X_{k}$ being the blow-up of $Z_{k}$ in $\mathbf{P}^{n}$. 
On $X$, we now have Cartier divisors $D_{k}$ (which are the inverse images of the $Z_{k}$ ) and line bundles $\mathcal{O}_{X}\left(D_{k}\right)$ equipped with a canonical section $s_{D_{k}} \in \Gamma\left(X, \mathcal{O}_{X}\left(D_{k}\right)\right)$. Moreover, $s_{D_{k}}$ and $\mathcal{O}_{X}\left(D_{k}\right)$ are pull backs of similar objects on $X_{k}$ (which we will denote by the same letters). Note also that $\mathcal{O}_{X}\left(-D_{k}\right)=\mathcal{I}_{Z_{k}} \cdot \mathcal{O}_{X} \subset \mathcal{O}_{X}$ and that by definition, $s_{D_{k}}$ is obtained by dualizing the pull back of the canonical inclusion $\mathcal{I}_{Z_{k}} \rightarrow \mathcal{O}_{\mathbf{P}^{n}}$.

Let $D_{0}$ be the strict transform of $Z_{0}$ in $X$. We have a canonical isomorphism:

$$
\pi^{*} \mathcal{O}_{X}\left(Z_{0}\right) \simeq \mathcal{O}_{X}\left(D_{0}+\sum_{k=1}^{r} D_{k}\right)
$$

Denoting by $s_{D_{0}}$ the canonical section of $\mathcal{O}_{X}\left(D_{0}\right)$, the tensor product $\bigotimes_{k=0}^{r} s_{D_{k}}$ equals the pull back on $X$ of the canonical section of $\mathcal{O}\left(Z_{0}\right)$.

The $D_{k}$ 's $(0 \leqslant k \leqslant r)$ form a basis of the Picard group of $X$. We identify the anticanonical sheaf in these coordinates:

PROPOSITION 1.5. If $Z$ is smooth, then $X$ is a smooth variety and its anticanonical sheaf is given by

$$
\begin{aligned}
K_{X}^{-1} & =\pi^{*}\left((n+1) Z_{0}\right) \otimes \bigotimes_{k=1}^{r} \mathcal{O}\left(-\left(\operatorname{codim}\left(Z_{k}\right)-1\right) D_{k}\right) \\
& =\bigotimes_{k=0}^{r} \mathcal{O}\left(\left(2+\operatorname{dim}\left(Z_{k}\right)\right) D_{k}\right) .
\end{aligned}
$$

Proof. See Hartshorne [7], Ex. 8.5, p. 188.

\subsection{METRIZATIONS ON BLOW-UPS}

Let $S$ be the spectrum of a Dedekind ring (which will be the ring of integers in $F$, or a localization of it, or a completion) or the spectrum of a field which is equipped with a valuation. Let $X$ be a projective scheme over $S$. For a locally free sheaf $\mathcal{E}$ of finite rank on $X$, there are several notions of metrizations corresponding to these various cases. We recall briefly the definitions.

- If $S=\operatorname{Spec}(F)$, the spectrum of a field endowed with a valuation, a metric on $\mathcal{E}$ is a family of norms on the fibres $\mathcal{E}_{x}$ for $x \in X(\bar{F})$, which vary continuously with $x$ for the $F$-topology on $X(\bar{F})$.

- If $\bar{F}=\mathbf{C}$, one may ask that the dependence is $\mathcal{C}^{\infty}$, and -independently - that the metrics are Hermitian in the fibers.

- If $S=\operatorname{Spec}(F)$, where $F$ is the fraction field of a discrete valuation ring $R$, any flat and projective model $(\mathcal{X}, \mathcal{E})$ over $\operatorname{Spec}(R)$ determines a metric according to which a section is of norm $\leqslant 1$ at a point iff it is integral. 
- If $S=\operatorname{Spec}(F), F$ being a number field, an adelic metric on $\mathcal{E}$ is a collection of metrics for all $X_{v} / F_{v}, v$ being the different places of $F$. Moreover, one assumes that there exists a model over $\operatorname{Spec}\left(\mathfrak{b}_{F}\right)$ which gives the same metrics except at a finite number of places. At these exceptional places the ratios of the two metrics are assumed to be bounded functions on $X$.

The usual definitions of metrics on subsheafs, quotients, hom's, etc. are compatible with these notions.

Let $X$ be a quasi-projective flat scheme over $S, \mathcal{I}$ a sheaf of ideals on $X$ and $Z=V(\mathcal{I})$. Let $\pi: Y \rightarrow X$ be the blow-up of $V(\mathcal{I}), Y=\operatorname{Proj}\left(\bigoplus_{n} \mathcal{I}^{n}\right)$. On $Y$, the inverse image of $Z$ becomes a Cartier divisor $D$ and the line bundle $\mathcal{O}(D)$ is equipped with a canonical section $s_{D}$. We want to endow $\mathcal{O}_{Y}(D)$ with a metric and to give a formula for the norm of $s_{D}$ at any point of $Y \backslash|D|$. Note that $\mathcal{O}(-D)=\mathcal{I} \cdot \mathcal{O}_{Y} \subset \mathcal{O}_{Y}$ and that $s_{D}$ is the pull back of the canonical inclusion $\mathcal{I} \rightarrow \mathcal{O}_{X}$.

Choose a locally free sheaf $\mathcal{E}$ of finite rank on $X$ with a section $\sigma_{Z} \in \Gamma(X, \mathcal{E})$ whose scheme of zeroes is $Z$ (existence follows from the quasi-projectivity of $X$ ). This induces a surjective homomorphism $\phi: \mathcal{E}^{\vee} \rightarrow \mathcal{I}$ and a closed immersion $Y \hookrightarrow \mathbf{P}\left(\mathcal{E}^{\vee}\right)$ such that $\mathcal{O}(-D)=\mathcal{I} \cdot \mathcal{O}_{Y}=\mathcal{O}_{\mathbf{P}}(1)$ and the universal quotient map $\pi^{*} \mathcal{E}^{\vee} \rightarrow \mathcal{O}_{\mathbf{P}}(1)$ on $Y$ is the pull back of $\phi$. Hence, to metrize $\mathcal{O}_{Y}(D)$ it is sufficient to endow $\mathcal{E}^{\vee}$ with a metric. The quotient metric on $\mathcal{O}_{\mathbf{P}}(1)$ is defined as follows: for any local section $s$ of $\mathcal{O}_{\mathbf{P}}(1)$, we pose $\|s\|(y)=\inf _{t}\|t\|(y)$, where the infimum is on the local sections $t$ of $\pi^{*} \mathcal{E}^{\vee}$ mapping to $s$ under the canonical surjection $\pi^{*} \phi: \pi^{*} \mathcal{E}^{\vee} \rightarrow \mathcal{O}_{\mathbf{P}}(1)$

Restrict this to $Y$. This gives a norm on $\left.\mathcal{O}_{\mathbf{P}}(1)\right|_{Y}=\mathcal{O}_{Y}(-D)$. The dual norm on $\mathcal{O}_{Y}(D)$ of the canonical section $\mathrm{s}_{D}$ is given by the formula

$$
\left\|\mathrm{s}_{D}\right\|=\sup _{s \neq 0} \frac{\left|\left\langle\mathbf{s}_{D}, s\right\rangle\right|}{\|s\|}=\sup _{t} \frac{\left|\left\langle\mathrm{s}_{D}, \pi^{*} \phi(t)\right\rangle\right|}{\|t\|}
$$

the last supremum being over the non-zero local sections $t$ of $\pi^{*} \mathcal{E}^{\vee}$. But, away from $D$ on the blow-up, $t$ comes from a local section of $\mathcal{E}^{\vee}$ and $\left\langle\mathbf{s}_{D}, \pi^{*} \phi(t)\right\rangle$ is exactly the image of $t$ under the surjection $\phi: \mathcal{E}^{\vee} \rightarrow \mathcal{I}$. Hence, $\left\|s_{D}\right\|$ is equal to the norm of $\phi$, viewed as a homomorphism $\mathcal{E}^{\vee} \rightarrow \mathcal{O}_{X}$, which by the definition of the dual norm on $\mathcal{E}^{\vee}$ is exactly the norm of the original section $\sigma_{Z} \in \Gamma(X, \mathcal{E})$.

Note the precise meaning of these calculations:

- they are valid if $S$ is any field with a valuation;

- if $S$ is a discrete valuation ring, arithmetic intersection on the integral model gives a result which is compatible with the metrized theory on the generic fibre if the metric on $\mathcal{E}$ comes from the model; 
- if $S$ is the ring of integers of a number field, they show that we have an adelic metric in the sense of Arakelov geometry provided $\mathcal{E}$ is equipped with an adelic metric.

Hence, we have the following theorem:

THEOREM 1.7. Let $X$ be an algebraic variety over a field $F, \mathcal{I} \subset \mathcal{O}_{X}$ a sheaf of ideals on $X$ and $\pi: Y \rightarrow X$ the blow-up of $\mathcal{Y}$. Let $\mathcal{E}$ be a locally free sheaf of finite rank on $X$ with a section $\sigma_{Z} \in \Gamma(X, \mathcal{E})$ such that $V(\mathcal{I})=\operatorname{div}\left(\sigma_{Z}\right)$ as schemes.

Assume $\mathcal{E}$ is given a metric. Then the line sheaf $\mathcal{O}_{Y}(D)$ corresponding to the exceptional divisor $D$ on $Y$ has a canonical metric such that the norm of its canonical section $s_{D}$ at a point $y \in Y$ is given by the formula:

$$
\left\|s_{D}\right\|(y)=\left\|\sigma_{Z}\right\|(\pi(y)) \text {. }
$$

In particular, if $\mathcal{L}_{1}, \ldots, \mathcal{L}_{r}$ are line bundles on $X$ with sections $s_{i}$ such that, as a scheme, $Z=\bigcap \operatorname{div}\left(s_{i}\right)$, we may take $\mathcal{E}=\bigoplus \mathcal{L}_{i}, \sigma_{Z}=\left(s_{i}\right)$. Assume the $\mathcal{L}_{i}$ to be metrized and endow $\mathcal{E}$ with the associated Hermitian metric (resp. with the $\ell^{\infty}$-metric at non-Archimedean places). The preceding theorem implies that $\mathcal{O}_{Y}(D)$ may be metrized in such a way that

$$
\left\|\boldsymbol{s}_{D}\right\|^{2}(y)=\sum_{i=1}^{r}\left\|\boldsymbol{s}_{i}\right\|^{2}(\pi(y)) .
$$

In particular, if $X=\mathbf{P}^{n}, \mathcal{L}_{i}=\mathcal{O}_{\mathbf{P}^{n}}\left(n_{i}\right), \mathrm{s}_{i}$ corresponds to a homogeneous polynomial $g_{i}$ of degree $n_{i}$ and, if $\pi(y)=\left(x_{0}: \cdots: x_{n}\right)$,

$$
\left\|\boldsymbol{s}_{D}\right\|^{2}(y)=\sum_{i=1}^{r} \frac{\left|g_{i}\left(x_{0}, \ldots, x_{n}\right)\right|^{2}}{\left(\sum_{j=0}^{n}\left|x_{i}\right|^{2}\right)^{n_{i}}}
$$

As a last example, assume that $X=\mathbf{P}^{n}$ and $Z$ is an integral divisor in $Z_{0}$. Then, the homogeneous ideal of $Z$ is of the form $\left(x_{0}, f\left(x_{1}, \ldots, x_{n}\right)\right)$ for some homogeneous polynomial $f$ of degree $d \geqslant 1$. If $\pi(y)=\left(1: x_{1}: \cdots: x_{n}\right)$, then

$$
\left\|\boldsymbol{s}_{D}\right\|^{2}(y)=\frac{1}{1+\sum_{j=1}^{n}\left|x_{j}\right|^{2}}+\frac{\left|f\left(x_{1}, \ldots, x_{n}\right)\right|^{2}}{\left(1+\sum_{j=1}^{n}\left|x_{j}\right|^{2}\right)^{d}} .
$$

All these formulas have analogues at non-Archimedean places with the sum of the squares being replaced by their maximum.

\subsection{RÉSUMÉ}

Let $F$ be a number field. For $1 \leqslant k \leqslant r$, choose a finite family of homogeneous polynomials $g_{k, j} \in F\left[x_{0}, \ldots, x_{n}\right]$ of degree $d_{k, j}$ generating a prime ideal $\mathcal{I}_{Z_{k}}$ corresponding to an integral subscheme $Z_{k} \subset \mathbf{P}^{n}$. Let $\pi: X \rightarrow \mathbf{P}^{n}$ be the blow-up of 
the ideal $\mathcal{I}=\mathcal{I}_{Z_{1}} \cdots \mathcal{I}_{Z_{k}}$. On $X$, the inverse image of $Z_{k}$ is a Cartier divisor $D_{k}$ whose associated line bundle $\mathcal{O}_{X}\left(D_{k}\right)$ can be adelically metrized so that the norm of its canonical section $\mathbf{s}_{D_{k}}$ at a point $x \in X$ mapping to $\left(x_{0}: \cdots: x_{n}\right) \in \mathbf{P}^{n}$ is given by

$$
\left\|\mathrm{s}_{D_{k}}\right\|_{v}(x)=\max _{j} \frac{\left|g_{k, j}\left(x_{0}, \ldots, x_{n}\right)\right|_{v}}{\max \left(\left|x_{0}\right|_{v}, \ldots,\left|x_{n}\right|_{v}\right)^{d_{k, j}}}
$$

at finite places $v$, and by

$$
\left\|\mathrm{s}_{D_{k}}\right\|_{v}^{2}(x)=\sum_{j} \frac{\left|g_{k, j}\left(x_{0}, \ldots, x_{n}\right)\right|_{v}^{2}}{\left(\left|x_{0}\right|_{v}^{2}+\ldots+\left|x_{n}\right|_{v}^{2}\right)^{d_{k, j}}}
$$

if $v$ is an Archimedean place.

We shall henceforth assume that $Z_{k}$ is contained in the hyperplane at infinity $x_{0}=0$. Then one may assume that one of the $g_{k, j}=x_{0}$ and that the others do not depend on $x_{0}$. The universal property of the blow-up implies that $\pi: X \rightarrow \mathbf{P}^{n}$ is an isomorphism over $\mathbf{G}_{a}^{n} \simeq\left\{x_{0} \neq 0\right\}$ and that the action of $\mathbf{G}_{a}^{n}$ on $\mathbf{P}^{n}$ lifts to an action on $X$ and to an action on the line bundles $\mathcal{O}_{X}\left(D_{k}\right)$.

The following proposition can be deduced, either through explicit computations with the formulas defining $\left\|s_{D_{k}}\right\|$, or by an abstract argument involving schemes over Spec $\mathfrak{D}_{F}$.

PROPOSITION 1.13. Assume that the polynomials $g_{k, j}$ have coefficients in $\mathfrak{b}_{F}$ and that they generate the homogeneous ideal $\mathcal{I}_{Z_{k}} \cap \mathfrak{o}_{F}\left[x_{0}, \ldots, x_{n}\right]$.* Then, for each place $v$ of $F$, the standard compact subgroup $\mathbf{G}_{a}^{n}\left(\mathfrak{o}_{v}\right)$ of $\mathbf{G}_{a}^{n}\left(F_{v}\right)$ acts isometrically on $\mathcal{O}\left(D_{k}\right)$.

Let $D_{0}$ be the strict transform of the hyperplane at infinity under $\pi$. The line bundle $\mathcal{O}_{X}\left(D_{0}\right)$ is the pull back on $X$ of the $\mathcal{O}_{\mathbf{P}^{n}}(1)$ and we shall equip it with its standard metric (given by the formulas above, the family of $g_{0, j}$ being reduced to $x_{0}$ ). By means of Equations (1.4), (1.5), we then can metrize the line bundles $\mathcal{O}_{X}\left(D_{0}\right)$ and $K_{X}^{-1}$.

\section{Heights, Poisson Formula}

\subsection{PRODUCT FORMULA AND HEIGHTS}

We recall some conventions concerning absolute values in number fields.

Over $\mathbf{R}$, we set $|\cdot|_{\infty}$ to be the usual absolute value (such that $|2|_{\infty}=2$ !). If $p$ is a prime number, the absolute value over $\mathbf{Q}_{p}$ is normalized by $|p|_{p}=1 / p$. These absolute values extend uniquely to any algebraic extension of $\mathbf{R}$ or $\mathbf{Q}_{p}$.

If $F$ is a number field, we denote by $\operatorname{Val}(F)$ the set of places (equivalence classes of valuations) of $F$. If $v$ is a place of $F$, we will set $m_{v}=e_{v} f_{v}$, the product of the ramification index by the local degree at $v$ and we denote by $|\cdot|_{v}$ the $m_{v}$ th power

ॠThis means that the subscheme $V\left(\left(g_{k, j}\right)_{j}\right)$ of $\mathbf{P}_{\mathfrak{o}_{F}}^{n}$ is projective and flat over $\mathfrak{o}_{F}$. 
of the unique extension of $|\cdot|_{\infty}$ or $|\cdot|_{p}$ to $F_{v}$ (according to $v$ being archimedian or not). Now, for any $x \in F$ and any valuation $v$ of $\mathbf{Q}$,

$$
\prod_{w \mid v}|x|_{w}=\left|N_{F / \mathbf{Q}}(x)\right|_{v} .
$$

With these normalizations, we have the product formula: for any $x \in F^{*}$,

$$
\prod_{v \in \operatorname{Val}(F)}|x|_{v}=\prod_{v \in \operatorname{Val}(\mathbf{Q})}\left|N_{F / \mathbf{Q}}(x)\right|_{v}=1 .
$$

Let $X$ be a projective variety over $F$ and $\mathcal{L}$ a metrized line bundle on $X$. For any $x \in X(F)$, the (exponential, absolute) height of $x$ with respect to the metrized line bundle $\mathcal{L}$ is defined by

$$
H_{\mathcal{L}}(x)=\prod_{v \in \operatorname{Val}(F)}\|\mathbf{s}\|_{v}(x)
$$

where $s$ is any $F$-rational local section of $\mathcal{L}$, defined and nonzero at $x$. The product formula implies that the height doesn't depend on the choice of $s$.

\subsection{HEIGHTS ON BLOW-UPS}

We keep the notations of the preceding section. Moreover, we identify $\mathbf{G}_{a}^{n}$ with its isomorphic inverse image in $X$ under the blow-up $\pi: X \rightarrow \mathbf{P}^{n}$.

The metrizations above allow us to define height functions corresponding to complexified divisors $D(\mathbf{s})=s_{0} D_{0}+\ldots+s_{r} D_{r}$. Namely, if $v$ is a place of $F$ and $x=\left(x_{1}, \ldots, x_{n}\right) \in \mathbf{G}_{a}^{n}\left(F_{v}\right)$, its exponential local height is defined by

$$
H_{D(\mathbf{s}), v}(x)=\prod_{k=0}^{r}\left\|s_{D_{k}}\right\|_{v}^{-s_{k}}\left(1: x_{1}: \cdots: x_{n}\right)
$$

The global height of a point $x \in \mathbf{G}_{a}^{n}\left(\mathbf{A}_{F}\right)$ is then the product of all local heights. This gives a pairing

$$
H: \operatorname{Pic}^{G}(X)_{\mathbf{C}} \times \mathbf{G}_{a}^{n}\left(\mathbf{A}_{F}\right) \longrightarrow \mathbf{C}^{*}
$$

which is multiplicative as a function on $\operatorname{Pic}^{G}(X)$ and which is invariant under the action of the compact subgroup $\prod_{\text {finite } v} \mathbf{G}_{a}^{n}\left(\mathfrak{o}_{v}\right)$ of $\mathbf{G}_{a}^{n}\left(\mathbf{A}_{F}\right)$. Such a pairing had already appeared in the context of toric varieties.

The invariance of the heights is a crucial technical ingredient in the proofs of analytic properties of the height zeta functions for toric varieties and for equivariant compactifications of $\mathbf{G}_{a}^{n}$ considered in the present paper.

The 'height zeta function' is the series

$$
Z\left(s_{0}, \ldots, s_{r}\right)=\sum_{x \in \mathbf{G}_{a}^{n}(F)} H_{D(\mathbf{s})}(x)^{-1} .
$$


Its convergence in some nonempty open subset of $\mathbf{C}^{r+1}$ is a consequence of the following (well known) lemma.

LEMMA 2.3 (compare [1], Prop. 1.4). Let $V$ be a projective variety over a number field $F$ and $\left(\mathcal{L}_{i}\right)_{1 \leqslant i \leqslant d}$ a finite number of ample metrized line bundles on $V$. For $x \in V(F)$, define $H(\mathbf{s} ; x)=\prod_{i=1}^{d} H_{\mathcal{L}_{i}}(x)^{s_{i}}$. Then there exists an open nonempty subset $\Omega$ of $\mathbf{R}^{d}$ such that the series

$$
Z(\mathbf{s})=\sum_{x \in X(F)} H(\mathbf{s} ; x)^{-1}
$$

converges absolutely and uniformly for all $\mathbf{s} \in \mathbf{C}^{d}$ with $\operatorname{Re}(\mathbf{s})$ contained in $\Omega$.

Moreover, any other metrization on the $\mathcal{L}_{i}$ 's gives the same domain of convergence.

Proof. The usual proof of Northcott's theorem establishes a polynomial bound for the number of rational points of bounded exponential height. Hence, the height zeta function of $\left(\mathbf{P}^{n}, \mathcal{O}(1)\right)$ converges for $s \gg 0$. (There is no need to invoke Schanuel's theorem [19] which gives the precise asymptotics.)

Therefore, there are real numbers $\alpha_{i}$ such that $Z\left(0, \ldots, s_{i}, \ldots, 0\right)$ converges for $\operatorname{Re}\left(s_{i}\right) \geqslant \alpha_{i}$. Now, $Z(\mathbf{s})$ converges for any $\mathbf{s}=\left(s_{1}, \ldots, s_{d}\right) \in \mathbf{C}^{d}$ such that for each $i, \operatorname{Re}\left(s_{i}\right) \geqslant \alpha_{i}$.

\subsection{HARMONIC ANALYSIS ON THE ADDITIVE GROUP}

We recall basic facts concerning harmonic analysis on the group of adelic points $\mathbf{G}_{a}^{n}\left(\mathbf{A}_{F}\right)$ (cf., for example, [22]). For any prime number $p$, we can view $\mathbf{Q}_{p} / \mathbf{Z}_{p}$ as the $p$-Sylow subgroup of $\mathbf{Q} / \mathbf{Z}$. This allows us to define a local character $\psi_{p}$ of $\mathbf{G}_{a}\left(\mathbf{Q}_{p}\right)$ by setting

$$
\psi_{p}: x_{p} \mapsto \exp \left(2 \pi i x_{p}\right) .
$$

At the infinite place of $\mathbf{Q}$ we put

$$
\psi_{\infty}: x_{\infty} \mapsto \exp \left(-2 \pi i x_{\infty}\right)
$$

(here $x_{\infty}$ is viewed as an element in $\mathbf{R} / \mathbf{Z}$ ). The product of local characters gives a character $\psi$ of $\mathbf{G}_{a}\left(\mathbf{A}_{\mathbf{Q}}\right)$ and, by composition with the trace, a character of $\mathbf{G}_{a}\left(\mathbf{A}_{F}\right)$. For any $\mathbf{a} \in \mathbf{G}_{a}^{n}\left(\mathbf{A}_{F}\right)$ we obtain a character $\psi_{\mathbf{a}}$ of $\mathbf{G}_{a}^{n}\left(\mathbf{A}_{F}\right)$ by

$$
\mathbf{x} \mapsto \psi \circ \operatorname{tr}_{F / \mathbf{Q}}(\langle\mathbf{a}, \mathbf{x}\rangle)
$$

The choice of $\psi$ defines a self-duality of $\mathbf{G}_{a}^{n}\left(\mathbf{A}_{F}\right)$ (Pontryagin duality). For $v \in \operatorname{Val}(F)$, we denote by $\mu_{v}$ the standard normalized local Haar measures on $\mathbf{G}_{a}^{n}\left(F_{v}\right)$ and by $\mu=\prod_{v} \mu_{v}$ the self-dual measure on $\mathbf{G}_{a}^{n}\left(\mathbf{A}_{F}\right)$. The precise normalization can be found in (cf. [22] or [11], p. 280); for $F=\mathbf{Q}$, we have $\mu_{p}\left(\mathbf{Z}_{p}\right)=1$ and $\mu_{\infty}([0 ; 1])=1$. 
For a function $H$ on $\mathbf{G}_{a}^{n}\left(\mathbf{A}_{F}\right)$ we denote by $\hat{H}$ its Fourier-transform (with respect to the Haar measure $\mu$ )

$$
\hat{H}: \mathbf{G}_{a}^{n}\left(\mathbf{A}_{F}\right) \rightarrow \mathbf{C}, \quad \psi \mapsto \int_{\mathbf{G}_{a}^{n}\left(\mathbf{A}_{F}\right)} H(\mathbf{x}) \psi(\mathbf{x}) d \mu(\mathbf{x}),
$$

whenever the integral converges. We shall also use the notation $d \mathbf{x}$ for $d \mu(\mathbf{x})$.

We will use the Poisson formula in following form (cf. [11], p. 280).

THEOREM 2.5. Let $H$ be a continuous function on $\mathbf{G}_{a}^{n}\left(\mathbf{A}_{F}\right)$ such that both $H$ and $\hat{H}$ are integrable and such that the series $\sum_{\mathbf{a} \in \mathbf{G}_{(F)}^{n}} H(\mathbf{x}+\mathbf{a})$ converges absolutely and uniformly when $\mathbf{x}$ belongs to any compact subset in $\mathbf{G}_{a}^{n}\left(\mathbf{A}_{F}\right) / \mathbf{G}_{a}^{n}(F)$. Then, one has

$$
\sum_{\mathbf{x} \in \mathbf{G}_{a}^{n}(F)} H(\mathbf{x})=\sum_{\mathbf{a} \in \mathbf{G}_{a}^{n}(F)} \hat{H}\left(\psi_{\mathbf{a}}\right) .
$$

For $\mathbf{s} \in \mathbf{C}^{r+1}$ and $\psi \in \mathbf{G}_{a}^{n}\left(\mathbf{A}_{F}\right)$, we shall denote by $\hat{H}(\mathbf{s} ; \psi)$ the Fourier transform of the height function $H(\mathbf{s} ; \cdot)^{-1}$ on $\mathbf{G}_{a}^{n}\left(\mathbf{A}_{F}\right)$ at the character $\psi$. It is the product of the local Fourier transforms of the functions $H_{v}(\mathbf{s} ; \cdot)^{-1}$ for all $v \in \operatorname{Val}(F)$.

PROPOSITION 2.6. With the above notations, for all characters $\psi$ which are nontrivial on the standard compact subgroup of $\mathbf{G}_{a}^{n}\left(\mathbf{A}_{F}\right)$, we have $\hat{H}(\mathbf{s} ; \psi)=0$.

Proof. This follows from the invariance of the height under the action of the standard compact subgroup, see Prop. 1.13.

Consequently, we have a formal identity for the height zeta function:

$$
Z(\mathbf{s})=\sum_{\mathbf{a} \in \mathbf{G}_{a}^{n}\left(\mathfrak{o}_{F}\right)} \hat{H}\left(\mathbf{s} ; \psi_{\mathbf{a}}\right) .
$$

The following lemma verifies the two hypotheses of the Poisson formula 2.5 concerning $H$.

LEMMA 2.8. There exists a basis $\left(e_{j}\right)$ of $\mathbf{C}^{r+1}$ such that for any $\mathbf{s} \in \mathbf{C}^{r+1}$ satisfying $\operatorname{Re}\left(\left\langle e_{j}, \mathbf{s}\right\rangle\right) \geqslant 1$ and for any compact subset $K$ of $\mathbf{G}_{a}^{n}\left(\mathbf{A}_{F}\right) / \mathbf{G}_{a}^{n}(F)$, the series

$$
\sum_{\mathbf{a} \in \mathbf{G}_{a}^{n}(F)} H(\mathbf{s} ; \mathbf{x}+\mathbf{a})^{-1}
$$

converges absolutely and uniformly for $\mathbf{x} \in K$.

Proof. Choose a basis $\left(e_{j}\right)$ of $\mathbf{C}^{r+1}$ such that any line bundle $D(\mathbf{s})$ for $\mathbf{s} \in \mathbf{Z}^{r+1}$ satisfying $\left\langle e_{j}, \mathbf{s}\right\rangle \geqslant 1$ is ample. This is possible because the vector space in $\mathbf{R}^{r+1}$ spanned by ample divisors contains an open cone. Hence, Lemma 2.3 implies the existence of a real $\alpha>0$ such that the series converges absolutely when $\mathbf{x}=0$, uniformly for all $\mathbf{s} \in \mathbf{C}^{r+1}$ such that $\operatorname{Re}\left(\left\langle e_{j}, \mathbf{s}\right\rangle\right) \geqslant \alpha$. 
For any $\mathbf{x}$, the function $H(\mathbf{s} ; \mathbf{x}+\cdot)$ is another height function for $D(\mathbf{s})$, called 'twisted height' in our paper [4], Section 2.4, esp. Proposition 2.4.3. As the convergence of the height zeta function doesn't depend on metrizations, this implies the convergence for any $\mathbf{x}$. The uniformity for $\mathbf{x} \in K$ follows from the fact that the height functions can be mutually uniformly bounded.

Now, for the proof of the meromorphic continuation of the height zeta function it will be sufficient to prove that the $\hat{H}$-series on the right-hand side of equation (2.7), $1^{\mathrm{O}}$ ) converges for some $\operatorname{Re}\left(s_{0}\right)>\alpha, \operatorname{Re}\left(s_{0}-s_{k}\right)>\alpha$ big enough, and $2^{\mathrm{O}}$ ) continues meromorphically.

\subsection{INTEGRABILITY OF LOCAL HEIGHT FUNCTIONS}

The aim of this section is to prove a general result concerning the integrability of local height functions against a measure with singularities.

PROPOSITION 2.10. Let $X$ be a proper smooth variety of dimension d over a field $F_{v}$ which is a finite extension of $\mathbf{R}$ or $\mathbf{Q}_{p}$. Fix a finite number of metrized line bundles $\mathcal{L}_{\alpha}$ on $X$ together with sections $\mathbf{s}_{\alpha}$. Assume that their divisors $\operatorname{div}\left(\mathbf{s}_{\alpha}\right)$ are smooth and that their union is a divisor $D$ with normal crossings and let $U=X \backslash D$. Finally, let $\omega \in \Gamma\left(U, \Omega_{X / F_{v}}^{d}\right)$ be a meromorphic differential form of top degree. We assume that there are integers $\lambda_{\alpha}$ such that the divisor of $\omega$ equals $\sum_{\alpha} \lambda_{\alpha} \operatorname{div}\left(s_{\alpha}\right)$. Denote by $d \omega$ the associated measure on $U\left(F_{v}\right)$.

Then, the integral $\int_{U\left(F_{v}\right)} \prod_{\alpha}\left\|\mathbf{s}_{\alpha}\right\|^{r_{\alpha}}(x) d \omega$ converges if and only if for all $\alpha$, $r_{\alpha}>\lambda_{\alpha}-1$.

Proof. Using a partition of unity on $X$ for the $F_{v}$-topology, we may assume that $X$ is a relatively compact open subset $\Omega \subset F_{v}^{d}$, with local coordinates $x_{1}, \ldots, x_{d}$ and that the divisor $\sum_{\alpha} \operatorname{div}\left(s_{\alpha}\right)$ is given by the equation $x_{1} \ldots x_{a}=0$ for some integer $0 \leqslant a \leqslant d$. The integral is then

$$
I_{\Omega}=\int_{\Omega} \prod_{i=1}^{a}\left|x_{i}\right|_{v}^{\left(r_{\alpha(i)}-\lambda_{\alpha(i)}\right)} \exp \left(\sum_{\alpha} h_{\alpha}(x)\right) d x_{1} \ldots d x_{d}
$$

for some functions $h_{\alpha}$ giving the metrics in our local trivialization and which are therefore continuous and bounded.

Remark that the integral of $|x|_{v}^{s}$ over the unit ball of $F_{v}$ converges if and only if $s>-1$. The Fubini theorem shows that the integral $I_{\Omega}$ converges if and only if for each $i \in\{1, \ldots, a\}, r_{\alpha(i)}-\lambda_{\alpha(i)}>-1$. As any $\alpha$ appears in some chart, the proposition is proved.

\subsection{THE LOCAL FOURIER TRANSFORM IN THE ARCHIMEDEAN CASE}

When $F_{v}=\mathbf{R}$ or $\mathbf{C}$, we want to show that the local Fourier transform of the height function as a function of $\psi_{\mathbf{a}}$ decreases rapidly when the norm of $\mathbf{a} \in F_{v}^{n}$ grows 
to infinity. The proof proceeds by integration by parts, which requires some estimates.

LEMMA 2.12. Let $X$ be a smooth projective variety over $F_{v}$ and $Z$ be a smooth closed subscheme of $X$. Let $\partial$ be a global section of $\left(\Omega_{X}^{1}\right)^{\vee} \otimes \mathcal{I}_{Z}$, i.e. a derivation on $X$ vanishing on $Z$. Denote by $\pi: Y \rightarrow X$ the blow-up of $\mathcal{I}_{Z}$.

(1) Then the derivation $\left.\partial\right|_{\pi^{-1}(X \backslash Z)}$ extends uniquely to a derivation on $Y$.

(2) Let $\mathcal{E}$ be a vector bundle on $X$ equipped with a smooth Hermitian metric and $\mathrm{s}$ a global section of $\mathcal{E}$ whose divisor is $Z$. Then the function $\partial \log \|s\|$ extends uniquely to a smooth function on $Y$.

Proof. Choose local analytic coordinates on $X$ such that $Z$ is defined by $x_{1}=\cdots=x_{a}=0$. Then, $Y$ may be embedded in $\mathbf{P}^{a-1} \times \mathbf{A}^{d}$ with coordinates $\left(\left(t_{1}: \cdots: t_{a}\right),\left(x_{1}, \ldots, x_{d}\right)\right)$ and is given there by the equations $t_{i} x_{j}=t_{j} x_{i}$ for $i, j \in\{1, \ldots, a\}$. We consider the chart $t_{a} \neq 0$. Then, local coordinates on $Y$ are $t_{1}, t_{2}, \ldots, t_{a-1}, x_{a}, x_{a+1}, \ldots, x_{d}$ and $\pi: Y \rightarrow X$ is given by $x_{i}=t_{i} x_{a}$ if $i<a$.

On $X$, the derivation $\partial$ has the form

$$
\partial=\sum_{i=1}^{d} h_{i} \frac{\partial}{\partial x_{i}}
$$

for some functions $h_{i} \in\left(x_{1}, \ldots, x_{a}\right)$. Now, we have to verify that if $i<a, \partial t_{i}$ is a regular function on $Y$. But

$$
\partial t_{i}=\partial\left(x_{i} / x_{a}\right)=h_{i}(x) \frac{1}{x_{a}}-h_{a}(x) \frac{x_{i}}{x_{a}^{2}}=\frac{1}{x_{a}}\left(h_{i}(x)-h_{a}(x) t_{i}\right) \in \mathcal{O}_{Y}
$$

since

$$
h_{j}(x) \in\left(t_{1} x_{a}, \ldots, t_{a-1} x_{a}, x_{a}\right)=\left(x_{a}\right) .
$$

For the statement concerning norms, we may fix the coordinates so that $\|s\|^{2}(x)=\sum_{i=1}^{a}\left|x_{i}\right|^{2}$. Then,

$$
\begin{aligned}
\partial \log \|\mathbf{s}\|^{2} & =\frac{1}{\left|x_{1}\right|^{2}+\cdots+\left|x_{a}\right|^{2}}\left(\sum_{i=1}^{a} 2 x_{i} h_{i}(x)\right) \\
& =\frac{1}{\left|t_{1}\right|^{2}+\cdots+\left|t_{a}-1\right|^{2}+1}\left(\sum_{i=1}^{a} 2 t_{i} \frac{h_{i}(x)}{x_{a}}\right)
\end{aligned}
$$

is regular on $Y$.

PROPOSITION 2.13. For any compact subset $K \subset \mathbf{R}^{r+1}$ over which the function $H_{v}(\mathbf{s} ; \cdot)^{-1}$ is integrable, and for any integer $d \geqslant 1$, there exists a constant $c(d, K)$ such 
that for any $\mathbf{a} \in \mathbf{C}^{n}$ and any $\mathbf{s} \in \mathbf{C}^{r+1}$ with $\operatorname{Re}(\mathbf{s}) \in K$,

$$
\left|\hat{H}_{v}\left(\mathbf{s} ; \psi_{\mathbf{a}}\right)\right| \leqslant c(d, K)\left(\frac{1+\|\Im(\mathbf{s})\|}{1+\|\mathbf{a}\|}\right)^{d} .
$$

Proof. The 2 preceding lemmas imply that for any multiindex $\alpha \in \mathbf{N}^{n}$, the derivative $\frac{\partial^{\alpha}}{\partial x^{\alpha}}\left(\log \left\|s_{D}\right\|\right)(x)$ is bounded on $\mathbf{G}_{a}^{n}\left(F_{v}\right)$. Moreover, $\left\|s_{D}\right\|$ tends to 0 at infinity. We thus may integrate by parts $d$-times.

\section{Projective Space}

From now on, we work over the field of rational numbers $\mathbf{Q}$. It will be clear from the proofs that the case of general number fields is indeed similar.

This section is included to illustrate our approach in the simplest example: we give yet another proof of asymptotics for the number of rational points of bounded height on the standard projective space $\mathbf{P}^{n}$ over the field of rational numbers with the standard metrization of the line bundle $\mathcal{O}(1)$ given by the model $\mathbf{P}_{\mathbf{Z}}^{n}$ at the finite places and by the $L^{2}$-norms at the archimedian places.

To keep this section as self-contained as possible, we reprove the estimates needed without referring to the general estimates of the preceding section.

We will denote by $\mathbf{A}$ the ring of adèles $\mathbf{A}_{\mathbf{Q}}$, by $p$ a prime number. We have the normalized valuations $|\cdot|_{p}$ with $|p|_{p}=p^{-1}$ and the usual absolute value $|\cdot|_{\infty}$. If $\mathbf{a} \in \mathbf{G}_{a}^{n}(\mathbf{A})$, we denote by $\psi_{\mathbf{a}}$ the corresponding character via the identification of $\mathbf{G}_{a}^{n}(\mathbf{A})$ with its Pontryagin dual.

We are interested in the height zeta function

$$
Z(s)=\sum_{\mathbf{x} \in \mathbf{G}_{a}^{n}(\mathbf{Q})} H(\mathbf{x})^{-s}
$$

where $H(\mathbf{x})=H_{\infty}(\mathbf{x}) \prod_{p} H_{p}(\mathbf{x})$ with

$$
H_{v}(\mathbf{x}):=\|\mathbf{x}\|_{v}= \begin{cases}\left(1+\sum_{j=1}^{n}\left|x_{j}\right|_{v}^{2}\right)^{1 / 2} & \text { if } v \mid \infty \\ \max \left(1, \max _{j}\left|x_{j}\right|_{v}\right) & \text { if } v \text { is finite. }\end{cases}
$$

The series (3.1) converges absolutely and uniformly to a holomorphic function for $\operatorname{Re}(s) \gg 0$. For all $s$ such that both sides converge, we have the Poisson-formula identity (cf. 2.5)

$$
Z(s)=\sum_{\psi_{\mathbf{a}}} \hat{H}\left(s ; \psi_{\mathbf{a}}\right)
$$

This identity is the starting point for a meromorphic continuation of $Z(s)$. We now compute (resp. estimate) the local Fourier transforms. 
LEMMA 3.3. Let $p$ be a prime number. For all $s$ with $\operatorname{Re}(s)>n, H_{p}(s ; \cdot)$ is integrable on $\mathbf{Q}_{p}^{n}$ and its Fourier transform at the trivial character $\psi_{0}$ is given by

$$
\hat{H}_{p}\left(s ; \psi_{0}\right)=\frac{1-p^{-s}}{1-p^{-(s-n)}} .
$$

Proof. We decompose the domain of integration $\mathbf{Q}_{p}^{n}$ into subdomains

$$
U(\alpha)=\left\{\mathbf{x}=\left(x_{1}, \ldots, x_{n}\right) ;\|\mathbf{x}\|_{p}=p^{\alpha}\right\}
$$

for $\alpha \geqslant 1$ and

$$
U(0)=\left\{\mathbf{x}=\left(x_{1}, \ldots, x_{n}\right) ;\|\mathbf{x}\|_{p} \leqslant 1\right\}
$$

Then

$$
\begin{aligned}
\hat{H}_{p}\left(s ; \psi_{0}\right) & =\int_{U(0)} H(\mathbf{x})^{-s} \mathrm{~d} \mathbf{x}+\sum_{\alpha \geqslant 1} \int_{U(\alpha)} H(\mathbf{x})^{-s} \mathrm{~d} \mathbf{x} \\
& =1+\sum_{\alpha \geqslant 1} p^{-\alpha s} \cdot \operatorname{vol}(U(\alpha)) .
\end{aligned}
$$

One has $\operatorname{vol} U(0)=1$ and for $\alpha \geqslant 1$,

$$
\operatorname{vol}(U(\alpha))=p^{\alpha n} \operatorname{vol}\left(\mathbf{Z}_{p}^{n} \backslash\left(p \mathbf{Z}_{p}\right)^{n}\right)=p^{\alpha n}\left(1-p^{-n}\right)
$$

For all $s$ with $\operatorname{Re}(s)>n$, the geometric series converges absolutely and we obtain

$$
\begin{aligned}
\hat{H}_{p}\left(s ; \psi_{0}\right) & =1+\left(1-\frac{1}{p^{n}}\right) \sum_{\alpha \geqslant 1} p^{-\alpha(s-n)}, \\
& =1+\left(1-\frac{1}{p^{n}}\right) \cdot \frac{1}{p^{s-n}} \cdot \frac{1}{1-p^{-(s-n)}} .
\end{aligned}
$$

Simplifying, we obtain (3.4).

For all $\mathbf{a}=\left(a_{1}, \ldots, a_{n}\right) \in \mathbf{Z}^{n}$ let $S(\mathbf{a})$ be the set of all primes which divide all $a_{j}$.

LEMMA 3.5. For all $\mathbf{a} \in \mathbf{Z}^{n} \backslash\{0\}$, all $s$ with $\operatorname{Re}(s)>n$ and all $p \notin S(\mathbf{a})$ we have

$$
\hat{H}_{p}\left(s ; \psi_{\mathbf{a}}\right)=1-p^{-s}
$$

Proof. As above, we have

$$
\hat{H}_{p}\left(s, \psi_{\mathbf{a}}\right)=1+\sum_{\alpha \geqslant 1} p^{-\alpha s} \int_{U(\alpha)} \psi_{\mathbf{a}}(\mathbf{x}) d \mathbf{x} .
$$


We first integrate over the set $V(\alpha)$ of $\mathbf{x} \in \mathbf{Q}_{p}^{n}$ such that $\|\mathbf{x}\| \leqslant p^{\alpha}$.

$$
\int_{V(\alpha)} \psi_{\mathbf{a}}(\mathbf{x}) d \mathbf{x}=p^{\alpha n} \int_{\mathbf{Z}_{p}^{n}} \psi_{\mathbf{a} / p^{\alpha}}(\mathbf{x}) d \mathbf{x} .
$$

If $\alpha \geqslant 1$, as $p$ doesn't divide all the $a_{j}$, this is the integral of a nontrivial character on a compact group, hence 0 . For $\alpha=0$, we get 1 . Therefore, as $V(0)=U(0)$ and $U(\alpha)=V(\alpha) \backslash V(\alpha-1)$ for $\alpha \geqslant 1$,

$$
\int_{U(\alpha)} \psi_{\mathbf{a}}(\mathbf{x}) d \mathbf{x}= \begin{cases}0 & \text { for } \alpha \geqslant 2 \\ -1 & \text { for } \alpha=1\end{cases}
$$

This implies the lemma.

LEMMA 3.7. For all $\varepsilon>0$ there exist constants $c$ and $\delta>0$ such that for all $s$ with $\operatorname{Re}(s)>n+\varepsilon$ and all $\mathbf{a} \in \mathbf{Z}^{n} \backslash\{0\}$ we have the estimate

$$
\left|\prod_{p \in S(\mathbf{a})} \hat{H}_{p}\left(s ; \psi_{\mathbf{a}}\right)\right| \leqslant c \cdot(1+\|\mathbf{a}\|)^{\delta} .
$$

Proof. In the integral, we replace $\psi_{\mathbf{a}}$ by $1, s$ by $\operatorname{Re}(s)$ and use the computation in (3.3). For $\operatorname{Re}(s) \geqslant n+\varepsilon$, we obtain

$$
\left|\hat{H}_{p}\left(s ; \psi_{\mathbf{a}}\right)\right| \ll \frac{1}{1-p^{-\varepsilon}} .
$$

If $a$ is a positive integer, we have the inequality

$$
\sum_{p \mid a} \frac{1}{p^{\varepsilon}} \ll \ln (1+a)
$$

This gives us equation (3.8).

We now turn to the estimations of the local Fourier transform for the place at infinity. For the trivial character we can compute the integral explicitly:

LEMMA 3.10. For all $s$ with $\operatorname{Re}(s)>n, H_{\infty}(s ; \cdot)$ is integrable on $\mathbf{R}^{n}$ and its Fourier transform at the trivial character $\psi_{0}$ is given by

$$
\hat{H}_{\infty}\left(s ; \psi_{0}\right)=\pi^{n / 2} \frac{\Gamma((s-n) / 2)}{\Gamma(s / 2)} .
$$

LEMMA 3.11. For all $\delta>0$ and all compacts $K$ in the domain $\operatorname{Re}(s)>n$ there exists $a$ constant $c(\delta, K)$ such that for all $\mathbf{a} \in \mathbf{Z}^{n}$ and all $s \in K$ we have

$$
\left|\hat{H}_{\infty}\left(s ; \psi_{\mathbf{a}}\right)\right| \leqslant c(\delta, K)(1+|\Im(s)|)^{\delta}(1+\|\mathbf{a}\|)^{-\delta}
$$


Proof. By a unitary change of variables, we may assume that $\mathbf{a}=(\|\mathbf{a}\|, 0, \ldots, 0)$. Thus,

$$
\begin{aligned}
\hat{H}_{\infty}\left(s ; \psi_{\mathbf{a}}\right)= & \int_{\mathbf{R}^{n}}\left(1+\|x\|^{2}\right)^{-s / 2} \exp \left(-2 \pi i\|\mathbf{a}\| x_{1}\right) \mathrm{d} \mathbf{x} \\
= & \int_{\mathbf{R}} \int_{\mathbf{R}^{n-1}}\left(1+\left|x_{1}\right|^{2}+\left\|\mathbf{x}^{\prime}\right\|\right)^{-s / 2} \exp \left(-2 \pi i\|a\| x_{1}\right) \mathrm{d} x_{1} \mathrm{~d} \mathbf{x}^{\prime} \\
= & \int_{\mathbf{R}}\left(1+\left|x_{1}\right|^{2}\right)^{-(s-n+1) / 2} \exp \left(-2 \pi i\|a\| x_{1}\right) \mathrm{d} x_{1} \times \\
& \times \int_{\mathbf{R}^{n-1}} \frac{\mathrm{d} \mathbf{y}}{\left(1+\|\mathbf{y}\|^{2}\right)^{s / 2}}
\end{aligned}
$$

For any $k>0$, the $k$ th derivative of $t \rightarrow\left(1+t^{2}\right)^{-s}$ is of the form $P_{k}(t)\left(1+t^{2}\right)^{-s-k}$ with $P_{k}$ a polynomial of degree $k$ whose coefficients are polynomials in $s$. Thus we can integrate by parts and get for any $k$ an expression

$$
\begin{gathered}
\int_{\mathbf{R}}\left(1+t^{2}\right)^{-(s-n+1) / 2} \exp (-2 \pi i\|a\| t) d t \\
=\frac{1}{(\pi i\|a\|)^{k}} \int_{\mathbf{R}} \frac{P_{k}(t)}{\left(1+t^{2}\right)^{k-\frac{s-n+1}{2}}} d t
\end{gathered}
$$

which imply the lemma.

Remark. It follows from the arguments above that the Fourier transform has polynomial growth in vertical strips.

THEOREM 3.12. The series

$$
Z(s)=\sum_{\psi_{\mathbf{a}}} \hat{H}\left(s ; \psi_{\mathbf{a}}\right)
$$

converges absolutely and uniformly to a holomorphic function for $s$ with $\operatorname{Re}(s)>n+1$. The function $Z(s)$ admits a meromorphic continuation to the domain $\operatorname{Re}(s)>n$ with exactly one simple pole at $s=n+1$. The residue at this pole equals

$$
\lim _{s \rightarrow n+1}(s-(n+1)) \hat{H}\left(s ; \psi_{0}\right)=\lim _{s \rightarrow n+1}(s-(n+1)) \int_{\mathbf{G}_{a}^{n}\left(\mathbf{A}_{\mathbf{Q}}\right)} H(s ; \mathbf{x}) d \mathbf{x} .
$$

Proof. Choose a real number $\delta>n$. From the lemmas above, it follows that there exists $\delta>0$ such that for any compact $K \in] n ;+\infty\left[\right.$, any $\mathbf{a} \in \mathbf{Z}^{n} \backslash\{0\}$, and any real $\delta^{\prime}>0$, the product of the local Fourier transforms at the character $\psi_{\mathbf{a}}$ converges to a holomorphic function of $s$ which satisfies the inequality

$$
\left|\hat{H}\left(s ; \psi_{\mathbf{a}}\right)\right| \leqslant c(K)(1+|\Im(s)|)^{\delta+\delta^{\prime}}(1+\|a\|)^{-\delta^{\prime}}, \quad \operatorname{Re}(s) \in K .
$$


Hence, the sum over all nontrivial $\psi$ converges absolutely and locally uniformly to a holomorphic function in the domain $\operatorname{Re}(s)>n$.

At the trivial character, we have, if $\operatorname{Re}(s)>n+1$,

$$
\hat{H}\left(s ; \psi_{0}\right)=\frac{\zeta(s-n) \Gamma((s-n) / 2)}{\zeta(s) \Gamma(s / 2)} .
$$

This has a simple pole at $s=n$ and extends meromorphically to the domain $\operatorname{Re}(s)>n$, with no other pole there.

The identification of the residue and Peyre's Tamagawa constant in [13] is straightforward, granted the meromorphic continuation of $\hat{H}\left(s ; \psi_{0}\right)$.

\section{Blow-ups of $\mathbf{P}^{2}$}

\subsection{PRELIMINARIES}

We continue to work over $\mathbf{Q}$ and we keep the notations of previous sections.

Let us consider the projective plane $\mathbf{P}^{2}$ with coordinates $\left(x_{0}: x_{1}: x_{2}\right)$ and its Zariski open subset $U \subset \mathbf{P}^{2}$ given by $x_{0} \neq 0$. Denote by $X$ the blow-up of $\mathbf{P}^{2}$ in $r$ distinct points $Z_{1}, \ldots, Z_{r}$ contained in the line at infinity $Z_{0} \subset \mathbf{P}^{2}$ which is given by $x_{0}=0$.

For all $k \in\{1, \ldots, r\}$, there is a linear form $\ell_{k} \in \mathbf{Z}\left[x_{1}, x_{2}\right]$ with coprime coefficients such that $Z_{k}=\mathcal{V}\left(x_{0}, \ell_{k}\right)$. For $k=1, \ldots, r$, we denote by $D_{k}$ the inverse image of $Z_{k}$ in $X$ and by $D_{0}$ the strict transform of the line $Z_{0}$. The variety $X$ is smooth; the anticanonical class is given by

$$
\left[K_{X}^{-1}\right]=3\left[D_{0}\right]+2 \sum_{k=1}^{r}\left[D_{k}\right]
$$

In the sequel, we shall identify a point $\mathbf{x} \in \mathbf{G}_{a}^{2}$ with the point with homogeneous coordinates $(1: \mathbf{x})$ in $\mathbf{P}^{2}$ or with its pre-image in the blow-up. It follows from the general theory of height functions on blow-ups given in Section 1 that for all $k \in\{1, \ldots, r\}$, a local height function for the divisor $D_{k}$ at such a point $\mathbf{x}$ is given by

$$
H_{k, p}(\mathbf{x})=\frac{\max \left(1,\|\mathbf{x}\|_{p}\right)}{\max \left(1,\left|\ell_{k}(\mathbf{x})\right|_{p}\right)}
$$

at a finite place $p$, and by an analogous formula where $\max (1, \cdot)$ is replaced by $\sqrt{1+.^{2}}$ at the infinite place. For $D_{0}$, we have

$$
H_{0, p}(\mathbf{x})=\max \left(1,\|\mathbf{x}\|_{p}\right) \prod_{k=1}^{r} H_{k, p}^{-1}(\mathbf{x})
$$


(with the same convention if $v=\infty$ ). The global height is given by

$$
H_{k}(\mathbf{x})=H_{k, \infty}(\mathbf{x}) \cdot \prod_{p} H_{k, p}(\mathbf{x})
$$

and for $\mathbf{s}=\left(s_{0}, \ldots, s_{r}\right) \in \mathbf{C}^{r}$, we define

$$
H(\mathbf{s} ; \mathbf{x}):=\prod_{k=0}^{r} H_{k}(\mathbf{x})^{s_{k}}
$$

the global height corresponding to the complexified line bundle $D(\mathbf{s})$.

From 2.5, we see that the height zeta function for $X$ has the following formal 'Fourier expansion':

$$
\sum_{\mathbf{x} \in \mathbf{Q}^{2}} H(\mathbf{s} ; \mathbf{x})^{-1}=\sum_{\mathbf{a} \in \mathbf{Z}^{2}} \hat{H}\left(\mathbf{s} ; \psi_{\mathbf{a}}\right)
$$

We have the decomposition

$$
\hat{H}\left(\mathbf{s} ; \psi_{\mathbf{a}}\right)=\hat{H}_{\infty}\left(\mathbf{s} ; \psi_{\mathbf{a}, \infty}\right) \cdot \prod_{p} \hat{H}_{p}\left(\mathbf{s} ; \psi_{\mathbf{a}, p}\right) .
$$

As in the case of $\mathbf{P}^{n}$, we compute the local Fourier transforms for almost all places and estimate them at the remaining bad places.

Let $S$ be the set of primes of bad reduction of the schematic closure of $\bigcup_{k} Z_{k}$ in $\mathbf{P}_{\mathbf{Z}}^{2}$. A prime $p$ belongs to $S$ if there exist two linear forms $\ell_{k}$ and $\ell_{j}$ such that $p$ divides $\operatorname{det}\left(\ell_{k}, \ell_{j}\right)$.

\subsection{DECOMPOSITION OF THE DOMAIN}

Fix a prime $p \notin S$. We may omit $p$ from the notations for norms, etc. Define subsets of $\mathbf{Q}_{p}^{2}$ as follows:

- $U(0)=\mathbf{Z}_{p}^{2}$

- if $1 \leqslant \beta<\alpha$ and $k \in\{1, \ldots, r\}, U_{k}(\alpha, \beta)$ is the set of $\mathbf{x} \in \mathbf{Q}_{p}^{2}$ such that $\|\mathbf{x}\|=p^{\alpha}$ and $\left|\ell_{k}(\mathbf{x})\right|=p^{\alpha-\beta}$

- if $\alpha \geqslant 1$ and $k \in\{1, \ldots, r\}, U_{k}(\alpha)$ is the set of $\mathbf{x} \in \mathbf{Q}_{p}^{2}$ such that $\|\mathbf{x}\|=p^{\alpha}$ and $\left|\ell_{k}(\mathbf{x})\right| \leqslant 1$

- if $\alpha \geqslant 1, U(\alpha)$ is the set of $\mathbf{x} \in \mathbf{Q}_{p}^{2}$ such that $\|\mathbf{x}\|=p^{\alpha}$ and all $\left|\ell_{j}(\mathbf{x})\right|=p^{\alpha}$.

As $p \notin S$, these sets furnish a partition of $\mathbf{Q}_{p}^{2}$. This decomposition is well adjusted to our local heights since they are constant on each subset:

- on $U(0)$, all $H_{k}$ 's are 1 ;

- on $U_{k}(\alpha, \beta), H_{k}=p^{\beta}$, the other $H_{j}$ with $j \geqslant 1$ are 1 and $H_{0}=p^{\alpha-\beta}$;

- on $U_{k}(\alpha), H_{k}=p^{\alpha}$, the other $H_{j}$ are 1 ;

- on $U(\alpha), H_{0}=p^{\alpha}$ and all other are 1 . 
In other words,

$$
H(\mathbf{s} ; \mathbf{x})= \begin{cases}1 & \text { if } \mathbf{x} \in U(0) \\ p^{\alpha s_{0}} & \text { if } \mathbf{x} \in U(\alpha), \alpha \geqslant 1 ; \\ p^{\alpha s_{0}+\beta\left(s_{k}-s_{0}\right)} & \text { if } \mathbf{x} \in U_{k}(\alpha, \beta), 1 \leqslant \beta<\alpha \\ p^{\alpha s_{k}} & \text { if } \mathbf{x} \in U_{k}(\alpha) .\end{cases}
$$

Table I on p. 91 summarizes this information.

\subsection{SOME INTEGRALS OF CHARACTERS}

We identify $\mathbf{a} \in \mathbf{Z}^{2}$ with the linear form it defines on $\mathbf{G}_{a}^{2}$ as well as with the character $\psi_{\mathbf{a}}$ of $\mathbf{G}_{a}^{2}\left(\mathbf{A}_{\mathbf{Q}}\right)$ it determines.

We will say that a character is generic if $\mathbf{a}$ is not proportional to any $\ell_{k}$. A nontrivial character is special if it is proportional to some (necessarily unique) $\ell_{k}$.

If $\mathbf{a}$ is generic, $S(\mathbf{a})$ is the set of primes in $S$ and all primes such that $p$ divides some determinant $\operatorname{det}\left(\ell_{k}, \mathbf{a}\right)$.

If $\mathbf{a}$ is special for $\ell_{k}, S(\mathbf{a})$ is the set of primes in $S$ and all primes such that $p$ divides some determinant $\operatorname{det}\left(\ell_{j}, \mathbf{a}\right)$ for $j \neq k$.

In particular, if $p \mid \mathbf{a}$, then $p \in S(\mathbf{a})$.

We now compute the integral of $\psi_{\mathbf{a}}$ over the subsets defined in the previous subsection, at least in the cases when $p \notin S(\mathbf{a})$.

Remark that for any $\mathbf{a}, \int_{U(0)} \psi_{\mathbf{a}}(\mathbf{x}) d \mathbf{x}=1$.

LEMMA 4.5 (Trivial character). Let $p$ be a prime not in $S$. Then,

$$
\begin{aligned}
& \operatorname{vol} U_{k}(\alpha, \beta)=p^{2 \alpha-\beta} \frac{(p-1)^{2}}{p^{2}} ; \\
& \operatorname{vol} U_{k}(\alpha)=p^{\alpha} \frac{p-1}{p} ; \\
& \operatorname{vol} U(\alpha)=p^{2 \alpha} \frac{(p-1)(p+1-r)}{p^{2}}
\end{aligned}
$$

LEMMA 4.6 (Generic character). Let $\mathbf{a}$ be a generic character and $p \notin S(\mathbf{a})$. Then,

$$
\begin{aligned}
& \int_{U_{k}(\alpha, \beta)} \psi_{\mathbf{a}}=0 ; \\
& \int_{U_{k}(\alpha)} \psi_{\mathbf{a}}= \begin{cases}-1 & \text { if } \alpha=1, \\
0 & \text { else } ;\end{cases} \\
& \int_{U(\alpha)} \psi_{\mathbf{a}}= \begin{cases}-1+r & \text { if } \alpha=1, \\
0 & \text { else; }\end{cases}
\end{aligned}
$$


LEMMA 4.7 (Special character). Let $\mathbf{a}$ a character which is special for $\ell_{k}$. If $p \notin S(\mathbf{a})$ and $j \neq k$, one has

$$
\begin{aligned}
& \int_{U_{j}(\alpha, \beta)} \psi_{\mathbf{a}}=0 ; \\
& \int_{U_{j}(\alpha)} \psi_{\mathbf{a}}= \begin{cases}-1 & \text { if } \alpha=1, \\
0 & \text { else; }\end{cases} \\
& \int_{U_{k}(\alpha, \beta)} \psi_{\mathbf{a}}= \begin{cases}-p^{\alpha} \frac{p-1}{p} & \text { if } \beta=\alpha-1, \\
0 & \text { else; }\end{cases} \\
& \int_{U_{k}(\alpha)} \psi_{\mathbf{a}}=p^{\alpha} \frac{p-1}{p} ; \\
& \int_{U(\alpha)} \psi_{\mathbf{a}}= \begin{cases}-(p+1-r) & \text { if } \alpha=1, \\
0 & \text { else; }\end{cases}
\end{aligned}
$$

Proof. We prove the three lemmas simultaneously. By a unitary change of variables, we may assume that $\ell_{k}(\mathbf{x})=x_{1}$. Then one has

$$
U_{k}(\alpha, \beta)=p^{\beta-\alpha} \mathbf{Z}_{p}^{*} \times p^{-\alpha} \mathbf{Z}_{p}^{*}
$$

and

$$
U_{k}(\alpha)=\mathbf{Z}_{p} \times p^{-\alpha} \mathbf{Z}_{p}^{*},
$$

hence their volumes are as in formulas (4.5a) and (4.5b).

If $p$ does not divide $\operatorname{det}\left(\ell_{k}, \mathbf{a}\right)$, we may change variables and even assume that $\mathbf{a}=(0,1)$. Then,

$$
\int_{U_{k}(\alpha, \beta)} \psi_{\mathbf{a}}(\mathbf{x}) d \mathbf{x}=p^{2 \alpha-\beta} \frac{p-1}{p} \int_{\mathbf{Z}_{p}^{*}} \exp \left(2 \pi i u / p^{\alpha}\right) d u
$$

and the last integral has already been calculated when we studied the case of $\mathbf{P}^{n}$ (end of proof of Lemma 3.5): one finds 0 if $\alpha \geqslant 2$ and $-1 / p$ if $\alpha=1$. But $\alpha>\beta \geqslant 1$, so $\alpha \neq 1$. This proves formulas (4.6a) and (4.7a).

Similarly,

$$
\int_{U_{k}(\alpha)} \psi_{\mathbf{a}}(\mathbf{x}) d \mathbf{x}=p^{\alpha} \int_{\mathbf{Z}_{p}^{*}} \exp \left(2 \pi i u / p^{\alpha}\right) d u
$$

is -1 for $\alpha=1$ and 0 else. Formulas (4.6b) and (4.7b) are therefore proved.

We now treat the case of a character a which is special for $\ell_{k}$. A unitary change of variables allows to assume $\ell_{k}(\mathbf{x})=x_{1}$ and $\mathbf{a}=(1,0)$. Then,

$$
\int_{U_{k}(\alpha, \beta)} \psi_{\mathbf{a}}(\mathbf{x}) d \mathbf{x}=p^{2 \alpha-\beta} \frac{p-1}{p} \int_{\mathbf{Z}_{p}^{*}} \exp \left(2 \pi i x / p^{\alpha-\beta}\right)
$$


is 0 if $\alpha-\beta \neq 1$ and is equal to

$$
p^{2 \alpha-\alpha+1} \frac{p-1}{p} \frac{(-1)}{p}=-p^{\alpha} \frac{p-1}{p}
$$

if $\beta=\alpha-1$, as stated in (4.7c). Equation (4.7d) follows from

$$
\int_{U_{k}(\alpha)} \psi_{\mathbf{a}}(\mathbf{x}) d \mathbf{x}=\int_{\mathbf{Z}_{p} \times p^{-\alpha} \mathbf{Z}_{p}^{*}} \exp \left(2 \pi i x_{1}\right) d \mathbf{x}=p^{\alpha} \frac{p-1}{p} .
$$

To compute the volume of $U(\alpha)$, it is useful to remark that $U(\alpha)$ is $p^{-\alpha}$ times the complementary subset in $\mathbf{Z}_{p}^{2}$ of $1+(p-1) r$ disjoint balls of radius $1 / p$. Therefore,

$$
\operatorname{vol} U(\alpha)=p^{2 \alpha}\left(1-\frac{1+(p-1) r}{p^{2}}\right)=p^{2 \alpha} \frac{(p-1)(p+1-r)}{p^{2}},
$$

as in formula $(4.5 \mathrm{c})$.

If $p \nmid \mathbf{a}$, remark that the integral of $\psi_{\mathbf{a}}$ over $p^{-\alpha}\left(\mathbf{Z}_{p}^{2} \backslash p \mathbf{Z}_{p}^{2}\right)$ is -1 for $\alpha=1$ and 0 for $\alpha \geqslant 2$. We now need to subtract the integrals over the disjoint subsets $U_{k}(\alpha, \beta)$ and $U_{k}(\alpha)$.

For a generic character, one gets 0 if $\alpha \geqslant 2$ and $-1+r$ if $\alpha=1$; this establishes formula (4.6c). Finally, if a is special for $\ell_{k}$, one has

$$
\int_{U(1)} \psi_{\mathbf{a}}(\mathbf{x}) d \mathbf{x}=-1+(r-1)-(p-1)=-(p+1-r)
$$

and $\int_{U(\alpha)} \psi_{\mathbf{a}}=0$ for $\alpha \geqslant 2$, as claimed in (4.7e).

\subsection{THE LOCAL FOURIER TRANSFORM AT $\psi_{0}$}

We still assume $p \notin S$ and compute the local Fourier transform at the trivial character $\psi_{0}$. By the general result 2.10 on Fourier transforms of height functions, $H(\mathbf{s} ; \cdot)^{-1}$ is integrable on $\mathbf{G}_{a}^{n}\left(\mathbf{Q}_{p}\right)$ as soon as $\operatorname{Re}\left(s_{0}\right)>2$ and all $\operatorname{Re}\left(s_{k}\right)>1$. We then have:

$$
\begin{aligned}
\hat{H}_{p}\left(\mathbf{s} ; \psi_{0}\right)= & \int_{U(0)} H(\mathbf{s} ; \mathbf{x})^{-1} \mathrm{~d} \mathbf{x}+ \\
& +\left(\sum_{k=1}^{r} \sum_{1 \leqslant \beta<\alpha} \int_{U_{k}(\alpha, \beta)} H(\mathbf{s} ; \mathbf{x})^{-1} \mathrm{~d} \mathbf{x}+\sum_{\alpha=1}^{\infty} \int_{U_{k}(\alpha)} H(\mathbf{s} ; \mathbf{x})^{-1} \mathrm{~d} \mathbf{x}\right)+ \\
& +\sum_{\alpha=1}^{\infty} \int_{U(\alpha)} H(\mathbf{s} ; \mathbf{x})^{-1} \mathrm{~d} \mathbf{x}
\end{aligned}
$$

and we compute each sum separately. The integral over $U(0)$ is 1 . Now, for a fixed $k$, 
the integral over all $U_{k}(\alpha, \beta)$ is

$$
\begin{aligned}
\sum_{1 \leqslant \beta<\alpha} \int_{U_{k}(\alpha, \beta)} H(\mathbf{s} ; \mathbf{x})^{-1} \mathrm{~d} \mathbf{x} & =\frac{(p-1)^{2}}{p^{2}} \sum_{1 \leqslant \beta<\alpha} p^{-\alpha s_{0}} p^{-\beta\left(s_{k}-s_{0}\right)} p^{2 \alpha} p^{-\beta} \\
& =\frac{(p-1)^{2}}{p^{2}} \sum_{\beta=1}^{\infty} p^{-\beta\left(s_{k}-s_{0}+1\right)} \sum_{\alpha=\beta+1}^{\infty} p^{-\alpha\left(s_{0}-2\right)} \\
& =\frac{(p-1)^{2}}{p^{2}} \sum_{\beta=1}^{\infty} p^{-\beta\left(s_{k}-s_{0}+1\right)} p^{-\beta\left(s_{0}-2\right)} \frac{1}{p^{s_{0}-2}-1} \\
& =\frac{(p-1)^{2}}{p^{2}} \frac{1}{p^{s_{0}-2}-1} \sum_{\beta=1}^{\infty} p^{-\beta\left(s_{k}-1\right)} \\
& =\frac{(p-1)^{2}}{p^{2}} \frac{1}{p^{s_{0}-2}-1} \frac{1}{p^{s_{k}-1}-1} .
\end{aligned}
$$

The sum over all $U_{k}(\alpha)$ ( $k$ fixed) equals

$$
\sum_{\alpha=1}^{\infty} \int_{U_{k}(\alpha)} H(\mathbf{s} ; \mathbf{x})^{-1} d \mathbf{x}=\frac{p-1}{p} \sum_{\alpha=1}^{\infty} p^{-\alpha s_{k}} p^{\alpha}=\frac{p-1}{p} \frac{1}{p^{s_{k}-1}-1} .
$$

Finally, the sum over all $U(\alpha)$ is

$$
\begin{aligned}
\sum_{\alpha=1}^{\infty} \int_{U(\alpha)} H(\mathbf{s} ; \mathbf{x})^{-1} \mathrm{~d} \mathbf{x} & =\frac{(p-1)(p+1-r)}{p^{2}} \sum_{\alpha=1}^{\infty} p^{-\alpha s_{0}} p^{2 \alpha} \\
& =\frac{(p-1)(p+1-r)}{p^{2}} \frac{1}{p^{s_{0}-2}-1}
\end{aligned}
$$

Putting all this together, we have

$$
\begin{aligned}
\hat{H}_{p}\left(\mathbf{s} ; \psi_{0}\right)= & 1+\frac{p-1}{p^{2}} \frac{1}{p^{s_{0}-2}-1} \sum_{k=1}^{r} \frac{1}{p^{s_{k}-1}-1}\left((p-1)+p\left(p^{s_{0}-2}-1\right)\right)+ \\
& +\frac{(p-1)(p+1-r)}{p^{2}} \frac{1}{p^{s_{0}-2}-1} \\
= & 1+\frac{p-1}{p^{2}} \frac{p^{s_{0}-1}-1}{p^{s_{0}-2}-1} \sum_{k=1}^{r} \frac{1}{p^{s_{k}-1}-1}+ \\
& +\frac{(p-1)(p+1-r)}{p^{2}} \frac{1}{p^{s_{0}-2}-1} \\
= & 1+\frac{p-1}{p^{2}} \frac{1}{p^{s_{0}-2}-1}\left((p+1-r)+\sum_{k=1}^{r} \frac{p^{s_{0}-1}-1}{p^{s_{k}-1}-1}\right) \\
= & 1+\frac{p^{2}-1}{p^{s_{0}}-p^{2}}+\frac{p-1}{p^{s_{0}}-p^{2}} \sum_{k=1}^{r} \frac{p^{s_{0}-1}-p^{s_{k}-1}}{p^{s_{k}-1}-1} .
\end{aligned}
$$


We remark that if $\left(s_{0}, s_{1}, \ldots, s_{r}\right)=(3,2, \ldots, 2) s$, corresponding to the multiple $K_{X}^{-s}$ of the anticanonical class, this yields

$$
\hat{H}_{p}\left(K_{X}^{-s} ; \psi_{0}\right)=1+\frac{p^{2}-1}{p^{3 s}-p^{2}}+r \frac{(p-1)\left(p^{3 s}-p^{2 s}\right)}{\left(p^{3 s}-p^{2}\right)\left(p^{2 s}-p\right)} .
$$

In particular, for $K_{X}^{-1}$, we have

$$
\begin{aligned}
\hat{H}\left(K_{X}^{-1} ; \psi_{0}\right) & =1+\frac{p^{2}-1}{p^{3}-p^{2}}+r \frac{p-1}{p^{3}-p^{2}} \frac{p^{2}-p}{p-1} \\
& =1+\frac{r+1}{p}+\frac{1}{p^{2}}=\frac{1}{p^{2}} \# X\left(\mathbf{F}_{p}\right),
\end{aligned}
$$

the expected local density at $p$.

\subsection{THE LOCAL FOURIER TRANSFORM AT A GENERIC CHARACTER}

Let $\mathbf{a}$ be a generic character and $p \notin S(\mathbf{a})$. In that case, the summation is easier. The integrals over $U_{k}(\alpha, \beta)$ are 0 , as are the integrals over $U_{k}(\alpha)$ or $U(\alpha)$ if $\alpha \geqslant 2$. Therefore

$$
\hat{H}\left(\mathbf{s} ; \psi_{\mathbf{a}}\right)=1-\sum_{k=1}^{r} p^{-s_{k}}+(r-1) p^{-s_{0}} .
$$

For $K_{X}^{-s}$, this specializes to

$$
\hat{H}\left(K_{X}^{-s} ; \psi_{\mathbf{a}}\right)=1-r p^{-2 s}+(r-1) p^{-3 s} .
$$

\subsection{THE LOCAL FOURIER TRANSFORM AT A SPECIAL CHARACTER}

If $\mathbf{a}$ is special for $\ell_{k}$ and $p \notin S(\mathbf{a})$, it behaves as if it were generic for the other $\ell_{j}$. Besides $U(0), U(1)$ and $U_{j}(1)$ for $j \neq k$, remain the integrals over $U_{k}(\alpha, \alpha-1)$ for $\alpha \geqslant 2$ and the integrals over $U(\alpha)$ for $\alpha \geqslant 2$.

$$
\begin{aligned}
\hat{H}\left(\mathbf{s} ; \psi_{\mathbf{a}}\right)= & 1-\sum_{j \neq k} p^{-s_{j}}+(r-p-1) p^{-s_{0}}+ \\
& +\sum_{\alpha=1}^{\infty} p^{\alpha} \frac{p-1}{p} p^{-\alpha s_{k}}-\sum_{\alpha \geqslant 2} p^{\alpha} \frac{p-1}{p} p^{-\alpha s_{0}-(\alpha-1)\left(s_{k}-s_{0}\right)} \\
= & 1-\sum_{j \neq k} p^{-s_{j}}+(r-p-1) p^{-s_{0}}+ \\
& +\frac{p-1}{p} \frac{1}{p^{s_{k}-1}-1}-\frac{p-1}{p} p^{s_{k}-s_{0}} \frac{p^{-s_{k}+1}}{p^{s_{k}-1}-1} \\
= & 1-\sum_{j \neq k} p^{-s_{j}}+(r-p-1) p^{-s_{0}}+(p-1)\left(1-p^{1-s_{0}}\right) \frac{1}{p^{s_{k}}-p} .
\end{aligned}
$$


For $K_{X}^{-s}$, one has

$$
\hat{H}\left(K_{X}^{-s} ; \psi_{\mathbf{a}}\right)=1-(r-1) p^{-2 s}+(r-p-1) p^{-3 s}+(p-1) \frac{1-p^{1-3 s}}{p^{2 s}-1} .
$$

\subsection{BAD REDUCTION, GENERAL ESTIMATES}

If $p \in S(\mathbf{a})$, then the previous analysis doesn't say anything about the behaviour of $\hat{H}\left(\mathbf{s} ; \psi_{\mathbf{a}}\right)$. However, for any compact contained in the domain of integrability of the height function, there is a uniform estimate $\left|\hat{H}_{p}\left(\mathbf{s} ; \psi_{\mathbf{a}}\right)\right| \leqslant C$, where the constant $C$ doesn't depend on $p \in S$. For $p \in S(\mathbf{a}) \backslash S$, we replace $\psi_{\mathbf{a}}$ by 1 and insert the estimates for the trivial character. If $s_{0} \geqslant 3-\varepsilon$ and $s_{k} \geqslant 2-\varepsilon$ for $1 \leqslant k \leqslant r$, we have an estimate $\operatorname{bigl}\left|H_{p}\left(\mathbf{s} ; \psi_{\mathbf{a}}\right)-1\right| \leqslant c p^{-\varepsilon}$, hence

$$
\prod_{p \in S(\mathbf{a})}\left|\hat{H}_{p}\left(\mathbf{s} ; \psi_{\mathbf{a}}\right)\right| \leqslant C^{\# S} \prod_{p \in S(\mathbf{a}) \backslash S}\left(1+c p^{-\varepsilon}\right) \leqslant C^{\prime}(1+\|\mathbf{a}\|)^{\kappa}
$$

for some constant $C^{\prime}$ and some $\kappa>0$. Indeed, if $p \in S(\mathbf{a}) \backslash S$, then $p$ divides $\prod_{j} \operatorname{det}\left(\ell_{j}, \mathbf{a}\right)$, where $j$ goes through all integers in $\{1, \ldots, r\}$ if $\mathbf{a}$ is generic, and avoids $k$ if $\mathbf{a}$ is special for $\ell_{k}$. This is a polynomial expression in $\mathbf{a}$ and we conclude using inequality (3.9).

\subsection{MEROMORPHIC CONTINUATION}

We split the sum over all characters in $r+2$ parts: the trivial character is treated separately; then the generic characters; then the characters which are special for $\ell_{k}, k$ varying from 1 to $r$ :

$$
\sum_{\mathbf{a}} \hat{H}\left(\mathbf{s} ; \psi_{\mathbf{a}}\right)=\hat{H}\left(\mathbf{s} ; \psi_{0}\right)+\sum_{\mathbf{a} \text { generic }} \hat{H}\left(\mathbf{s} ; \psi_{\mathbf{a}}\right)+\sum_{k=1}^{r} \sum_{\mathbf{a} \text { special for } \ell_{\mathrm{k}}} \hat{H}\left(\mathbf{s} ; \psi_{\mathbf{a}}\right) .
$$

Let $Z_{0}, Z_{\text {gen }}$ and $Z_{k}$ (for $1 \leqslant k \leqslant r$ ) be the functions defined by the corresponding series. We will fix some $\varepsilon>0$ and consider only $\mathbf{s}$ in the open set $\Omega$ of $\mathbf{C}^{r+1}$ defined by the inequalities $\operatorname{Re}\left(s_{0}\right)>\frac{5}{2}+\varepsilon$ and $\operatorname{Re}\left(s_{k}\right)>\frac{3}{2}+\varepsilon$ for $1 \leqslant k \leqslant r$.

If $\mathbf{a}$ is a generic character, the formula (4.11) shows that the Euler product

$$
\prod_{p \notin S(\mathbf{a}) \cup\{\infty\}} \hat{H}_{p}\left(\mathbf{s} ; \psi_{\mathbf{a}}\right)
$$

converges absolutely to a holomorphic function in $\Omega$ which is bounded independently of a. Granting the estimates at infinity 2.13 and for the places of bad reduction $p \in S(\mathbf{a})$ given in Section 4.14, the functions $\hat{H}\left(\cdot ; \psi_{\mathbf{a}}\right)$ are holomorphic in this domain and satisfy for any $\kappa>0$ a uniform inequality

$$
\left|\hat{H}\left(\mathbf{s} ; \psi_{\mathbf{a}}\right)\right| \leqslant C(\kappa)(1+\|\mathbf{s}\|)^{v(\kappa)}(1+\|\mathbf{a}\|)^{-\kappa}
$$


for some constants $C(\kappa)$ and $v(\kappa)$. Choosing $\kappa>n$ ensures that $Z_{\text {gen }}$ extends to a holomorphic function in $\Omega$ which has polynomial growth in vertical strips.

If $\mathbf{a}$ is special for $\ell_{k}, p \notin S(\mathbf{a})$ and $\mathbf{s} \in \Omega$, we can estimate from formula (4.13)

$$
\hat{H}_{p}\left(\mathbf{s} ; \psi_{\mathbf{a}}\right)=\left(1+p^{1-s_{k}}\right)\left(1+\mathrm{O}\left(p^{-1-\varepsilon}\right)\right) .
$$

Using again the estimates for primes $p \in S(\mathbf{a})$ and $p=\infty$ imply that

$$
\zeta\left(s_{k}-1\right) \hat{H}\left(\mathbf{s} ; \psi_{\mathbf{a}}\right)=\prod_{p} \hat{H}_{p}\left(\mathbf{s} ; \psi_{\mathbf{a}}\right)\left(1-p^{1-s_{k}}\right)
$$

extends to a holomorphic function on $\Omega$, with the following growth property:

$$
\left|\zeta\left(s_{k}-1\right) \hat{H}\left(\mathbf{s} ; \psi_{\mathbf{a}}\right)\right| \leqslant C(\kappa)(1+\|\mathbf{s}\|)^{v(\kappa)}(1+\|\mathbf{a}\|)^{-\kappa} .
$$

If we choose $\kappa>1$, we get that there exists a holomorphic function $h_{k}$ on $\Omega$ which has polynomial growth in vertical strips such that $Z_{k}(\mathbf{s})=h_{k}(\mathbf{s}) \zeta\left(s_{k}-1\right)$.

And finally, for the trivial character, we have absolute convergence of the Euler product for $\operatorname{Re}\left(s_{0}\right)>3$ and $\operatorname{Re}\left(s_{k}\right)>2$ for $1 \leqslant k \leqslant r$. In $\Omega$, it follows from Equation (4.9) that for any $p \notin S$,

$$
\hat{H}_{p}\left(\mathbf{s} ; \psi_{0}\right)=\left(1+p^{2-s_{0}}\right) \prod_{k=1}^{r}\left(1+p^{1-s_{k}}\right)\left(1+O\left(p^{-1-\varepsilon}\right)\right) .
$$

In the same way as before, we deduce the existence of a holomorphic function $h_{0}$ on $\Omega$ which has polynomial growth in vertical strips such that

$$
Z_{0}(\mathbf{s})=h_{0}(\mathbf{s}) \zeta\left(s_{0}-2\right) \zeta\left(s_{1}-1\right) \cdots \zeta\left(s_{r}-1\right) .
$$

Moreover, $h_{0}$ is the product of an absolutely convergent Euler product and of a factor at infinity which doesn't vanish on $\Omega \cap \mathbf{R}^{r+1}$. Hence, $h_{0}$ doesn't vanish on $\Omega \cap \mathbf{R}^{r+1}$, and $h_{0}(3,2, \ldots, 2) \neq 0$.

Finally, we have proven the existence of holomorphic functions $h, h_{0}, \ldots, h_{r}$ on $\Omega$, which have polynomial growth in vertical strips, such that $Z$ has a meromorphic continuation given by

$$
\begin{aligned}
Z(\mathbf{s})= & h_{0}(\mathbf{s}) \zeta\left(s_{0}-2\right) \zeta\left(s_{1}-1\right) \cdots \zeta\left(s_{r}-1\right)+ \\
& +\sum_{k=1}^{r} h_{k}(\mathbf{s}) \zeta\left(s_{k}-1\right)+h(\mathbf{s}) .
\end{aligned}
$$

The standard estimates (due to Rademacher, see [17]) for the growth of the Riemann zeta function in vertical strips now imply that, away from poles, $Z(\mathbf{s})$ has polynomial growth in vertical strips.

Therefore, we have proven the following theorem:

THEOREM 4.16. The height zeta function $Z(\mathbf{s})$ converges in the domain $\operatorname{Re}\left(s_{0}\right)>3$, $\operatorname{Re}\left(s_{k}\right)>2$. There exists a holomorphic function $g$ in the domain $\operatorname{Re}\left(s_{0}\right)>\frac{5}{2}$, 
Table I

\begin{tabular}{|c|c|c|c|c|}
\hline & $U(0)$ & $U_{k}(\alpha, \beta)$ & $U_{k}(\alpha)$ & $U(\alpha)$ \\
\hline Volume & 1 & $p^{2 \alpha-\beta} \frac{(p-1)^{2}}{p^{2}}$ & $p^{\alpha} \frac{p-1}{p}$ & $p^{2 \alpha} \frac{(p-1)(p+1-r)}{p^{2}}$ \\
\hline$H_{0}$ & 1 & $p^{\alpha-\beta}$ & 1 & $p^{\alpha}$ \\
\hline$H_{j}(j \neq k)$ & 1 & 1 & 1 & 1 \\
\hline$H_{k}$ & 1 & $p^{\beta}$ & $p^{\alpha}$ & 1 \\
\hline$H(\mathbf{s} ; \cdot)$ & 1 & $p^{\alpha s_{0}+\beta\left(s_{k}-s_{0}\right)}$ & $p^{\alpha s_{k}}$ & $p^{\alpha S_{0}}$ \\
\hline \multicolumn{5}{|c|}{ Integrals of a generic character $\psi_{\mathbf{a}}$} \\
\hline$\alpha=1$ & 1 & & 1 & $-1+r$ \\
\hline$\alpha \geqslant 2$ & 1 & 0 & 0 & 0 \\
\hline \multicolumn{5}{|c|}{ Integrals of a character $\psi_{\mathbf{a}}$ special for $\ell_{j}, j \neq k$} \\
\hline$\alpha=1$ & 1 & & -1 & $-(p+1-r)$ \\
\hline$\alpha \geqslant 2$ & 1 & 0 & 0 & 0 \\
\hline \multicolumn{5}{|c|}{ Integrals of a character $\psi_{\mathrm{a}}$ special for $\ell_{k}$} \\
\hline$\overline{\alpha=1}$ & 1 & & $p^{\alpha} \frac{p-1}{p}$ & $(p+1-r)$ \\
\hline$\alpha \geqslant 2$ & 1 & & $p^{\alpha} \frac{p-1}{p}$ & 0 \\
\hline$\beta=\alpha-1$ & \multicolumn{3}{|c|}{$-p^{\alpha} \frac{p-1}{p}$} & \\
\hline$\beta<\alpha-1$ & \multicolumn{2}{|r|}{0} & & \\
\hline
\end{tabular}

$\operatorname{Re}\left(s_{k}\right)>\frac{3}{2}$ such that

$$
Z(\mathbf{s})=g(\mathbf{s}) \frac{1}{\left(s_{0}-3\right)\left(s_{1}-2\right) \ldots\left(s_{r}-2\right)}
$$

and $g\left(K_{X}^{-1}\right) \neq 0$. Moreover, $g$ has polynomial growth in vertical strips.

COROLLARY 4.17. There exists a polynomial $P_{X}$ of degree $r$ and a real number $\delta>0$ such that the number of points of $\mathbf{G}_{a}^{2}(\mathbf{Q}) \subset X(\mathbf{Q})$ of anticanonical height $\leqslant B$ satisfies

$$
N\left(\mathbf{G}_{a}^{2}, K_{X}^{-1}, B\right)=B P_{X}(\log B)+O\left(B^{1-\delta}\right)
$$

Moreover, if $\tau\left(K_{X}\right)$ denotes the Tamagawa number, the leading coefficient of $P_{X}$ is equal to

$$
\frac{1}{r !} \frac{\tau\left(K_{X}\right)}{3 \cdot 2^{r}},
$$

as predicted by Peyre's refinement of Manin's conjecture. 
Remarks. (1) Our theorem implies a similar asymptotic formula for arbitrary line bundles $\mathcal{L}$, provided their class belongs to the interior of the cone of effective divisors.

(2) It is curious to note that we now have asymptotics for blow-ups of $\mathbf{P}^{2}$ in any number of points on a line, and in three points in general position, while the asymptotics for blow-ups of $\mathbf{P}^{2}$ in 4 points in general position are still unknown.

(3) The (numerical) intersection pairing on $X$ is given by the following formulae whose proof we omit. If $j \neq k \in\{1, \ldots, r\}$, we have $D_{j} \cdot D_{k}=0$. For any $j \in\{1, \ldots, r\}, D_{0} \cdot D_{j}=1$ and $D_{0} \cdot D_{0}=1-r$. This implies that the degree of $K_{X}^{-1}$ on $D_{0}$ is $\left(3 D_{0}+2 D_{1}+\cdots+2 D_{r}\right) \cdot D_{0}=3(1-r)+2 r=3-r$. Similarly, the degree of $K_{X}^{-1}$ on $D_{j}$ is equal to 1 . Schanuel's theorem implies that if $1 \leqslant k \leqslant r$, the number of points of anticanonical height $\leqslant B$ located on $D_{k}(1 \leqslant k)$ grows as $B^{2}$, and that for $r \geqslant 3$, the anticanonical height is bounded on $D_{0}$. Therefore, the subvarieties $D_{0}, \ldots, D_{r}$ are really 'accumulating' rational points.

\section{Acknowledgements}

The work of the second author was partially supported by the NSA.

\section{References}

1. Batyrev, V. V. and Manin, Yu. I.: Sur le nombre de points rationnels de hauteur bornée des variétés algébriques, Math. Ann. 286 (1990), 27-43.

2. Batyrev, V. V. and Tschinkel, Yu.: Tamagawa numbers of polarized algebraic varieties, In: Nombre et répartition des points de hauteur bornée [14], pp. 299-340.

3. Birch, B. J.: Forms in many variables, Proc. London Math. Soc. 265A (1962), 245-263.

4. Chambert-Loir, A. and Tschinkel, Yu.: Torseurs arithmétiques et espaces fibrés, E-print, math.NT/9901006, 1999.

5. Davenport, H.: Cubic forms in sixteen variables, Proc. Roy. Soc. London Ser. A 272 (1963), 285-303.

6. Franke, J., Manin, Yu. I. and Tschinkel, Yu.: Rational points of bounded height on Fano varieties, Invent. Math. 95(2) (1989), 421-435.

7. Hartshorne, R.: Algebraic Geometry, Grad. Texts in Math. 52, Springer, New York, 1977.

8. Hassett, B. and Tschinkel, Yu.: Geometry of equivariant compactifications of $\mathbf{G}_{a}^{n}, 1999$.

9. Heath-Brown, R.: Cubic forms in 10 variables, Proc. London Math. Soc. 47(2) (1983), $225-257$.

10. Hooley, C.: On nonary cubic forms, J. Reine Angew. Math. 386 (1988), 32-98.

11. Manin, Yu. I. and Panchishkin, A.: Number Theory I. Introduction to Number Theory, Springer, Berlin, 1995.

12. Mumford, D., Fogarty, J. and Kirwan F.: Geometric Invariant Theory, Ergeb. Math. Grenzgeb. (3) 34, Springer, Berlin, 1994.

13. Peyre, E.: Hauteurs et mesures de Tamagawa sur les variétés de Fano, Duke Math. J. 79 (1995), 101-218.

14. Peyre, E.: Nombre et répartition des points de hauteur bornée, Astérisque, No. 251, 1998.

15. Peyre, E.: Terme principal de la fonction zêta des hauteurs et torseurs universels, in Nombre et répartition des points de hauteur bornée [14], pp. 259-298. 
16. Peyre, E.: Torseurs et méthode du cercle, Tech. report, Isaac Newton Institute for Mathematical Sciences, 1998.

17. Rademacher, H.: On the Phragmén-Lindelöf theorem and some applications, Math. Z. 72 (1959/60), 192-204.

18. Salberger, P.: Tamagawa measures on universal torsors and points of bounded height on Fano varieties, in Nombre et répartition des points de hauteur bornée [14], pp. 91-258.

19. Schanuel, S.: Heights in number fields, Bull. Soc. Math. France 107 (1979), 433-449.

20. Schmidt, W.: The density of integer points on homogeneous varieties, Acta Math. 154(3-4) (1985), 243-296.

21. Strauch, M. and Tschinkel, Yu.: Height zeta functions of toric bundles over flag varieties, Selecta Math. 5(3) (1999), 325-396.

22. Tate, J. T.: Fourier analysis in number fields, and Hecke's zeta-functions, Algebraic Number Theory (Proc. Instructional Conf., Brighton, 1965), Thompson, Washington, D.C., 1967, pp. 305-347. 